\title{
Les Pisidiens À Rhodes AuX ÉPOQUes HelléNisTiQue ET ROMAINE
}

\author{
Hadrien Bru \\ Université de Bourgogne-Franche-Comté - \\ Institut des Sciences et Techniques de l'Antiquité, Besançon*
}

\begin{abstract}
In the perspective of a complete external prosopography of the Pisidians in progress, this article presents a commented catalogue of 61 persons who lived on the island of Rhodos and in its Carian Peraia from the 3rd century BCE to the beginning of the Roman Imperial period. Concerning those slaves, mercenaries, artists, craftsmen or merchants, a historical context is provided, then remarks on their juristic, social and economical status. The evoked documentation is based on inscribed monuments - mainly funerary - and amphora stamps.
\end{abstract}

Keywords: Pisidians, Rhodos, epigraphy, prosopography, amphora stamps, slaves, mercenaries, sculptors, Hellenistic and Roman wars.

En matière de géographie historique et de sociologie culturelle, l'étude de la territorialisation d'une population ou d'un peuple apporte nécessairement beaucoup à l'historien en quête de la compréhension de phénomènes sociaux, politiques, religieux ou économiques replacés dans un ancien contexte, général, qui fut celui d'un cadre de vie. Il apparaît en outre qu'une étude de la déterritorialisation de cette même population ou de ce peuple nous renseigne également sur sa territorialisation, son mode de vie et son histoire, en rapport avec d'autres populations ou peuples installé(e)s en d'autres espaces. Cette approche favorise néanmoins les études s'intéressant aux échanges culturels, aux éventuelles formes d'acculturation(s) ayant découlé du déplacement d'individus dont la documentation écrite nous a laissé la trace. C'est ainsi qu'on peut suggérer l'utilité d'une prosopographie historique externe des Pisidiens, notamment hors d'Anatolie et sur la longue durée, d'une part en n'hésitant pas à remonter lorsque cela est possible à l'époque classique qui fut celle des impérialismes d'Athènes et de l'empire perse achéménide, d'autre part en soulignant les effets qu'eurent les changements d'échelle géopolitique,

* Je remercie vivement Edward Dąbrowa de m'avoir invité à cette contribution pour Electrum, ainsi qu'Alain Bresson (Université de Chicago) pour ses remarques « rhodiennes », et Alister Filippini (Università di Chieti - Pescara). 
politique et humaine induits par les projets politiques liés à l'imperium Romanum, dans le sillage de la conquête d'Alexandre puis des royaumes hellénistiques. Dans le cas d'espèce, nous nous intéresserons ici aux Pisidien(ne)s recensé(e)s sur la riche et puissante île de Rhodes ${ }^{1}$ et, à titre complémentaire, dans sa proche Pérée de Carie.

S'il est bien sûr possible de retrouver au sein des sources documentaires antiques des personnages attestés comme « Pisidiens », l'utilisation exclusive du critère onomastique ne convient en revanche pas pour une telle recherche d'individus, parce qu'on ne peut distinguer, sauf exception, les anthroponymes « pisidiens » des autres noms de personnes de tradition linguistique louvite/post-louvite que l'on découvre fréquemment en Isaurie, en Lycaonie, en Lycie, en Carie ou en Cilicie, comme l'a déjà montré Ladislav Zgusta. ${ }^{2}$ Dans la mesure où l'anthroponymie des Pisidiens appartient à un ancien fond linguistique anatolien propre aux populations résidant pour un bon nombre au Sud de la chaîne montagneuse du Taurus, de la Carie à la Cilicie, le « recensement » des Pisidiens doit donc passer, dans les recherches épigraphiques, par la mention de l'ethnique «Pisidien » ou « Pisidienne », en langue grecque ou latine. La spécification de l'origine civique de la personne, c'est-à-dire la mention écrite du nom de la cité, constitue également un critère de recherche épigraphique, dans une perspective historico-culturelle (et non ethnique) qui vise à étudier l'histoire d'un groupe socio-historique se désignant comme tel dans les sources épigraphiques (inscriptions surtout, mais aussi secondairement timbres amphoriques).

L'enquête dont nous proposons les résultats provisoires dans le cadre de la prosopographie externe complète des Pisidiens en cours se fonde sur une documentation épigraphique essentiellement constituée d'épitaphes, ${ }^{3}$ souvent laconiques, mais aussi de listes d'étrangers, de souscripteurs ou de décrets d'associations religieuses, très actives sur l'île de Rhodes. La chronologie, qui ne repose hélas souvent que sur une appréciation paléographique du ductus et du contexte documentaire, peu de textes étant précisément datés ou datables avec précision, est parfois malaisée à établir, mais on peut néanmoins affirmer avec certitude que la plupart des textes épigraphiques et les quelques timbres amphoriques connus entrent dans une fourchette s'étendant du III ${ }^{e} s$. av. J.-C. au I ${ }^{\text {er }}$ s. de notre ère. On trouvera en annexe un catalogue qui recense pour l'instant quelque 61 Pisidien(ne)s, et auquel nous renverrons. Cet inventaire et ce décompte autorisent déjà quelques statistiques et des remarques, lesquelles nous conduiront d'une part à expliquer et à périodiser la venue des Pisidien(ne)s à Rhodes, d'autre part à évaluer leurs statuts sociaux, économiques, politiques et juridiques à la lumière des sources épigraphiques.

Sur 61 personnes recensées avec certitude comme originaires de Pisidie à Rhodes, 53 sont des hommes (soit $86.9 \%$ ) et seulement 8 sont des femmes (soit 13.1\%) : la surreprésentation masculine n'étonne pas dans des sociétés résolument traditionnelles et patriarcales (qu'il s'agisse des Pisidiens ou des Grecs), mais Rhodes eut sans doute par ailleurs besoin d'une main d'œuvre masculine, notamment dans les chantiers navals ou la marine marchande, sans évoquer les mercenaires. La distribution des Pisidien(ne)s attesté(e)s sur l'île est intéressante à observer : sur les 61 individus, 44 sont attestés sur le

1 Sur les étrangers à Rhodes en général, voir Morelli 1956, 126-190, d'où Robert 1958, 289-290, n 357 ; Sacco 1980, 517-528, d'où Robert 1982, 359, n 244 ; Boyxen 2018.

2 Zgusta 1964.

3 Concernant les monuments funéraires rhodiens, voir Fraser 1977 ; Berges - Nollé 1996. 
territoire de la ville de Rhodos (soit 72.1\% de l'ensemble), capitale insulaire incontestée depuis le synoecisme de 408-407 av. J.-C. et grand port international ; 8 personnes sont attestées sur le territoire de Lindos, riche par ses activités navales, et prestigieuse grâce à son sanctuaire d'Athéna Lindia depuis l'époque archaïque et classique ; 3 attestations proviennent de Ialysos, très ancienne installation grecque remontant à la période postmycénienne mais devenue moins influente ensuite; le mont Atabyros, abritant le fameux sanctuaire de Zeus Atabyrios sis à l'Ouest de l'île dans l'arrière-pays de Kamiros, mais commun aux Rhodiens, apporte 2 attestations, alors que le site de Kamiros lui-même ne semble en livrer aucune ; enfin dans la proche Pérée rhodienne de Carie, on découvre 4 attestations, dont 3 à Tymnos et 1 à Kasara.

L'origine des Pisidien(ne)s telle que donnée par les inscriptions est également riche d'enseignements : sur 61 individus, on connaît pour 46 d'entre eux leur cité pisidienne d'origine (soit 75.4\%), alors que 15 sont simplement «Pisidien(ne)s » (soit 24.6\%). Seules 6 cités ou communautés pisidiennes sont mentionnées : une écrasante majorité provient de $\operatorname{Selge}^{4}$ ( 25 personnes sur 61 , soit $41 \%$ du total), ce qui confirme la puissance et l'influence de cette cité du Sud de la Pisidie, hellénisée très tôt et historiquement placée au premier rang régional par les historiens. ${ }^{5} \mathrm{C}^{9}$ est ensuite Termessos (la Grande) ${ }^{6}$ qui arrive au second rang de l'envoi de Pisidiens à Rhodes avec 11 attestations (soit 18\% de l'ensemble), forte de sa position de glacis montagneux proche de la plaine pamphylienne et d'une politique précocement favorable à Rome. La communauté des Oroandeis (ou Orondeis), ${ }^{7}$ originaire des confins de la Phrygie Parorée, de la Pisidie septentrionale, de la Lycaonie et de l'Isaurie, apporte pas moins de 6 attestations (soit 9.8\% de l'ensemble), ${ }^{8}$ un témoignage historique d'importance, nous le verrons. La cité pisidienne d'Etenna, ${ }^{9}$ très proche de la plaine pamphylienne et dont les ressortissants sont bien attestés ailleurs, notamment en Égypte, est représentée par 2 personnes, alors que Sagalassos et Erymna fournissent chacune 1 occurrence. Restent 15 individus (soit 24.6\%) se disant « Pisidien(ne)s » sans précision d'une cité d'origine, une donnée méritant commentaire. Dernier élément statistique global mais essentiel, concernant l'onomastique des Pisidien(ne)s de Rhodes : leurs anthroponymes sont à 90.2\% helléniques (soit 55 noms sur 61), une preuve tangible de leur hellénisation culturelle déjà constatée dans le Sud de l'Anatolie taurique $;^{10} 4$ ou 5 anthroponymes sur 61 sont néanmoins d'origine

4 Pour les deux premiers inventaires les plus complets, voir Morelli 1956, 169, et Nollé - Schindler 1991, 48, T43 et p. 51-59, T48-T70.

5 Strabon (XII.7.1) affirme ainsi que les Selgiens sont « les plus considérables des Pisidiens » (oi $\Sigma \varepsilon \lambda \gamma \varepsilon i ̃ \zeta$,

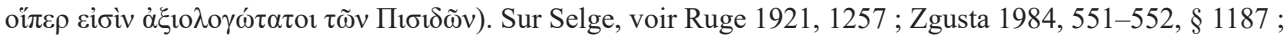
Nollé - Schindler 1991, 13-66 ; Bru 2017, 4, 29, 52, 223, 250-251, 254.

6 Pour un premier inventaire, cf. Morelli 1956, 173. Sur Termessos, voir Kruse 1934, 732-778 ; Zgusta 1984, 612-613, § 1320-3.

7 Pour un premier inventaire, cf. Morelli 1956, 164. Sur les Oroandeis, voir Ruge 1939, 1130-1132 ; Zgusta 1984, 445-447, § 946-1 ; Bru 2017, 27-28, 172-182.

8 Originaire de la plaine Killanienne située en Phrygie Parorée à proximité du tractus Orondicus et de la

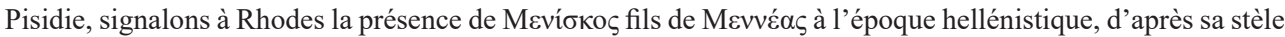
funéraire en marbre local découverte à Değirmen Dere (voir Maiuri 1925, 73, n 97 ; Robert 1938, 260-265 ;

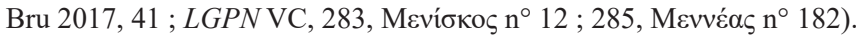

9 Bean 1970, 13-16 ; Zgusta 1984, 174-175, § 310 ; Nollé 1984, 142-156.

10 Mitchell 1991, 119-145. 
anatolienne $\mathrm{e}^{11}$ (soit de 6.5 à $8.2 \%$ ), et 1 d'origine iranienne ${ }^{12}$ (soit $1.6 \%$ ); aucun anthroponyme d'origine latine n'est recensé, ce qui confirme là encore les tendances observées en Pisidie, ${ }^{13}$ à corréler en outre avec la chronologie des inscriptions considérées, éminemment hellénistique à Rhodes.

Sur ce point, il importe de donner quelques précisions en vue d'affiner une esquisse de périodisation quant à la présence des Pisidien(ne)s sur l'île de Rhodes : 3 individus sont datés des III ${ }^{\mathrm{e}}-\mathrm{II}^{\mathrm{e}}$ s. av. J.-C. ; 12 au $\mathrm{II}^{\mathrm{e}}$ s. av. J.-C. $; 9$ aux II ${ }^{\mathrm{e}-\mathrm{I}^{\mathrm{er}}}$ s. av. J.-C. ; 11 au I ${ }^{\text {er }}$ s. av. J.-C. ; 5 aux I ${ }^{\text {er }}$ s. av. J.-C. - I ${ }^{\text {er }}$ s. ap. J.-C. ; 5 pour le I ${ }^{\text {er }}$ s. ap. J.-C. - époque impériale. Sachant que 7 attestations sont également attribuables à l'époque hellénistique de manière indéterminée, on en conclut donc qu'au moins 42 Pisidien(ne)s sur 61 sont présents sur l'île à l'époque hellénistique (soit 68.8\% de l'ensemble). Un peu davantage probablement, mais on retient que le «pic » de leur présence à Rhodes se situe sans aucun doute aux $\mathrm{II}^{\mathrm{e}}-\mathrm{I}^{\mathrm{er}}$ s. av. J.-C. ${ }^{14}$

Quels étaient leurs statuts juridiques et leur place socio-économique sur cette puissante île de marchands grecs ? À cela il n'est pas toujours aisé de répondre, en raison de textes souvent laconiques et funéraires en vertu des pratiques hellénistiques, sachant par exemple que parmi 61 Pisidien(ne)s, on ne connait qu'un seul patronyme, et que leurs professions ou conditions sociales ne sont pas explicitement spécifiées. Il est cependant possible d'esquisser quelques jalons sur la question.

La Pisidie, manifestement très populeuse et considérée comme «barbare » aux yeux de la plupart des Grecs, a fourni bon nombre d'esclaves à l'occasion de nombreuses guerres et expéditions régionales sur lesquelles nous reviendrons, surtout si l'on considère les rapports géographiquement et (géo)politiquement organiques qu'elle entretenait avec les cités pamphyliennes de Sidè, Attaleia, Aspendos ou Pergè, aux ports très actifs situés sur les littoraux méridionaux de l'Anatolie entre Rhodes et Chypre. Faute de précisions sur les monuments épigraphiques, c'est essentiellement l'onomastique qui permet de supposer le statut servile de certains individus dotés d'un anthroponyme caractéristique se rap-

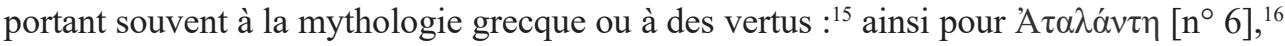
Selgienne de Rhodos dont le compagnon galate était "A $\theta \omega \varsigma$, sûrement esclave lui aussi

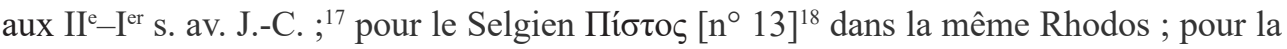

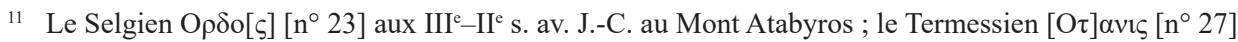

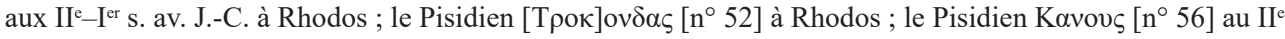
s. av. J.-C. à Ialysos ; et peut-être le Selgien $\Sigma \mathrm{PA} . \mathrm{O} \Sigma\left[\mathrm{n}^{\circ} 19\right]$ à Rhodos.

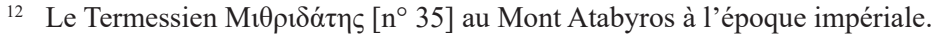

13 Voir par exemple pour le territoire de Tymbriada en Pisidie septentrionale, où l'on ne dénombre qu'un seul anthroponyme latin sur 104 (Bru 2017, 256).

14 Voir déjà Fraser 1977 à propos des monuments funéraires rhodiens en général, et Bresson 1997, 123 pour ce qui concerne les esclaves sur l'île.

15 Voir notamment Masson 1973, 9-23 ; Bresson 1997, 117-126.

16 Lambertz 1907, 24 ; Solin 1996, 349 (avec 10 attestations pour Atalante à Rome entre le Ire et le III ${ }^{\mathrm{e}}$ s. ap. J.-C.).

17 Notons qu'à Rome, H. Solin a répertorié Athus comme un nom d'esclave thrace au ${ }^{\text {er }} \mathrm{s}$. de notre ère (AE 1991, 260 ; Solin 1996, 609).

18 Lambertz 1908, 6; Solin 1996, 413 (avec 12 attestations pour Pistus à Rome entre le règne d'Auguste et le $\mathrm{III}^{\mathrm{e}}$ s. ap. J.-C.). 


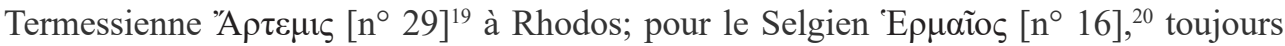

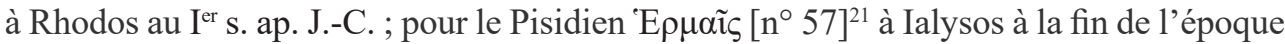

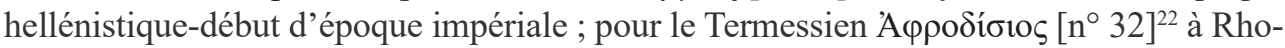

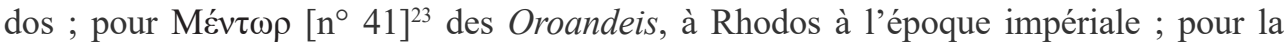

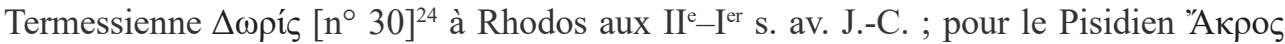
$\left[\mathrm{n}^{\circ} 55\right]^{25}$ à Rhodos à l'époque impériale; pour la Pisidienne Eưvol $\left.\alpha \mathrm{n}^{\circ} 58\right]^{26}$ à Ialysos ;

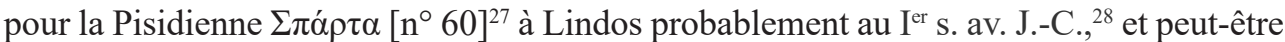

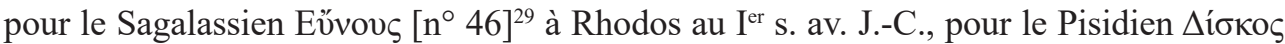
$\left[n^{\circ} 59\right]^{30}$ à Lindos aux III $-\mathrm{III}^{\mathrm{e}}$ s. av. J.-C., pour la Selgienne $\Phi \iota \lambda \omega^{\circ}\left[\mathrm{n}^{\circ} 5\right]^{31}$ à Rhodos au $\mathrm{II}^{\mathrm{e}}$ s. av. J.-C., pour le Selgien 'Ová $\sigma \mu \mathrm{s}\left[^{\circ} \mathrm{n}^{\circ}\right]^{32}$ au $\mathrm{II}^{\mathrm{e}}$ s. av. J.-C. à Rhodos ou pour le

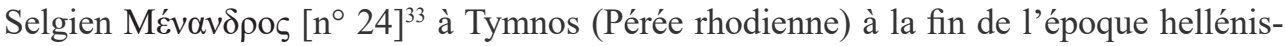
tique. D'autres esclaves sont probablement à ajouter, mais on note que dans l'hypothèse où les 16 individus précités seraient bien des esclaves, on parviendrait déjà à $25.8 \%$ de l'ensemble des Pisidiens recensés.

Au moins un fabricant d'amphores rhodiennes, ${ }^{34}$ si utilisées pour l'exportation du célèbre vin de l'île, est connu grâce aux timbres amphoriques du Selgien Máp $\omega v$ [ $\mathrm{n}^{\circ}$ 2] dont l'atelier céramique était actif à Rhodos entre 145 et 108 av. J.-C., alors qu'on inventorie également un anonyme de Selge [ $\left.\mathrm{n}^{\circ} 22\right]$ à Lindos. Précisons que le timbre

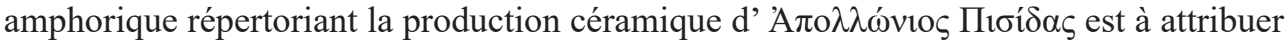

19 Lambertz 1907, 37 ; Solin 1996, 273 (avec 8 attestations pour Artemis à Rome entre le règne d'Auguste et le III ${ }^{\mathrm{e}}$ s. ap. J.-C.).

20 Lambertz 1907, 32 ; Solin 1996, 291 (avec 2 attestations à Rome aux Ir-II ${ }^{\mathrm{e}}$ s. ap. J.-C.).

21 Solin 1996, 291 recense 4 Hermais à Rome entre le $\mathrm{I}^{\text {er }}$ et le III ${ }^{\mathrm{e}}$ s. ap. J.-C., mais pour des femmes esclaves.

22 Lambertz 1907, 31 ; Solin 1996, 280-281 (avec 38 attestations pour Aphrodisius à Rome entre le $\mathrm{I}^{\mathrm{er}} \mathrm{s}$. av. J.-C. et la première moitié du $\mathrm{II}^{\mathrm{e}} \mathrm{s}$. de notre ère).

23 Solin 1996, 339 (avec 2 attestations pour Mentor à Rome entre le règne d'Auguste et le $\mathrm{I}^{\mathrm{er}}$ S. ap. J.-C.).

24 Lambertz 1907, 28 ; Solin 1996, 351 (avec 23 attestations pour Doris à Rome entre le règne d'Auguste et le III' s. ap. J.-C.).

25 Lambertz 1908, 7.

26 Solin 1996, 558 (avec 4 attestations pour Eunoea/Eunea/Eunia à Rome entre le règne d'Auguste et le III ${ }^{\mathrm{e}}$ s. ap. J.-C.).

27 Considérée à tort comme Pisidienne « unsicher » par Boyxen 2018, 382 (qui n’a pas corrigé « $\Sigma \tau$ qú $\rho \tau \alpha »$ suite à la lecture erronée de J. Hedenborg et A. Scrinzi donnant en deuxième lettre $\tau$ au lieu de $\pi$ ).

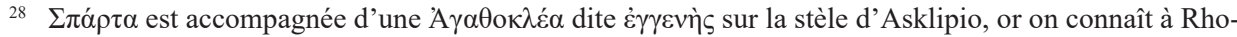

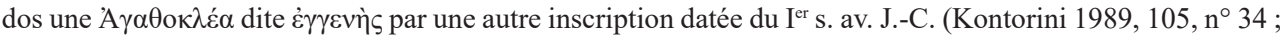
SEG 39, 822).

29 Lambertz 1908, 4 ; Solin 1996, 416 (avec 25 attestations pour Eunus à Rome entre le règne d'Auguste et le III ${ }^{\mathrm{e}}$ s. ap. J.-C.).

30 Lambertz 1908, 16 ; à Rome, on connaît pour des esclaves les noms de Disca au Irr s. ap. J.-C. (CIL VI, 12678) et $\operatorname{Disco}(n)$ aux I ${ }^{\text {er-II }}{ }^{\mathrm{e}}$ s. de notre ère (cf. Solin 1996, 540).

31 On connaît à Rome une vestale d'origine servile se nommant Philo (CIL VI, 5477 ; Solin 1996, 415).

32 Lambertz 1908, 5 ; Solin 1996, 465-467 (avec 185 attestations pour Onesimus à Rome entre le I ${ }^{\text {er }}$ s. av. J.-C. et le III ${ }^{\mathrm{e}} \mathrm{s}$. de notre ère).

33 Solin 1996, 259 (avec 35 attestations pour Menander à Rome entre la deuxième moitié du III ${ }^{\mathrm{e}} \mathrm{S}$. av. J.-C. et le III $^{\mathrm{e}} \mathrm{s}$. de notre ère).

34 Voir notamment Garlan 1998, 581-590. 
à l'atelier de Cnide, et non de Rhodes, ${ }^{35}$ sachant que ses amphores ont notamment été identifiées à Nea Paphos (Chypre) pour le deuxième quart du $\mathrm{II}^{\mathrm{e}}$ siècle avant notre ère. ${ }^{36}$ Lorsqu'ils étaient d'origine étrangère, ces importants artisans et commerçants semblent souvent avoir eu à Rhodes le statut de métèque.

Deux sculpteurs pisidiens sont connus grâce à leurs signatures à Rhodes : d'une part

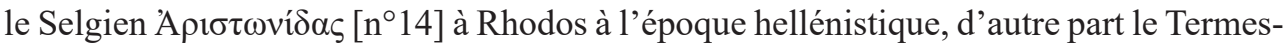
sien $\Phi i ́ \lambda \omega v$ [n $\left.{ }^{\circ} 28\right]$ actif dans la même cité entre 100 et 50 avant notre ère. Il est évident que la richesse commerciale des Rhodiens leur permit de multiplier les commandes à l'époque hellénistique, comme le prouve l'impressionnant inventaire de 106 signatures de sculpteurs rassemblées par Chr. Blinkenberg dans son volume consacré aux inscriptions de Lindos. ${ }^{37}$ Des artistes et artisans pisidiens faisant montre de leur technè se distinguèrent donc aussi dans ce domaine, probablement avec le statut de métèque.

Deux personnages originaires de Pisidie, dont un cité avec sa fille, sont explicitement désignés comme métèques par les inscriptions rhodiennes. Il s'agit tout d'abord du Sel-

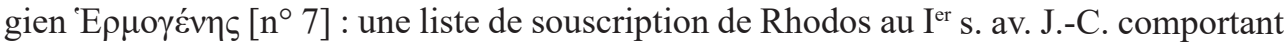
de nombreux étrangers montre qu'il a bellement contribué pour sa famille (notamment

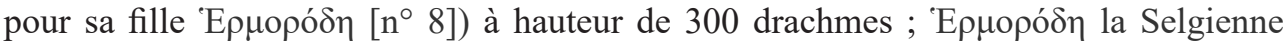
s'était visiblement mariée à un métèque originaire d'Ilion. Une urne cinéraire fragmen-

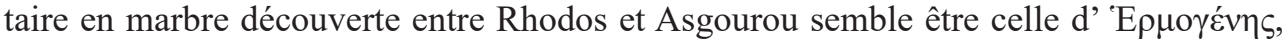
dans ce cas seul double témoignage épigraphique d'un Pisidien que nous paraissons posséder sur l'île. C'est la même liste de souscription précitée qui nous donne à Rhodos le nom d'un autre métèque cette fois originaire de la communauté des Oroandeis, Mñvis

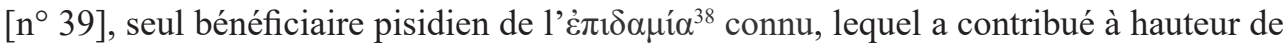
100 drachmes ; $\Delta \eta \mu \eta ́ \tau \rho ı \varsigma ~\left[n^{\circ} 40\right]$, également originaire de la communauté des Oroandeis, est lui aussi désigné comme métèque, au sein d'une liste qui paraît souvent répertorier des marchands résidant sur l'île.

Peu de Pisidiens peuvent être considérées comme des «notables » officiels à Rhodes,

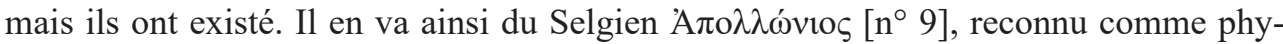

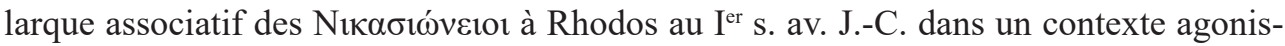
tique qui impliquait d'assez lourdes dépenses (l'inscription considérée cite agonothètes et gymnasiarques). Dans le même milieu social, riche et favorisé, se trouve égale-

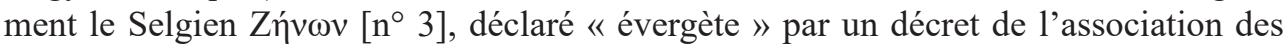

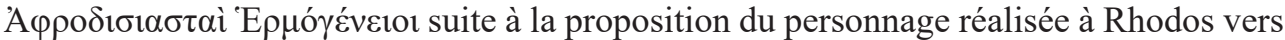
109-108 avant notre ère, sans que nous connaissions, comme souvent, la nature du ou des bienfait(s) en question. Vers la même époque, mais cette fois à Kasara dans la Pérée

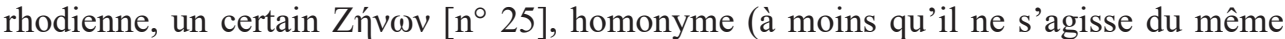
personnage que le précédent, sans certitude), lui aussi originaire de Selge, est honoré par une dédicace du koinon des Asklapiastes lui octroyant deux couronnes (feuilles et or),

35 Comme paraît l'entendre Boyxen 2018, 410, tab. 15, $\mathrm{n}^{\circ} 18$.

36 Voir Nicolaou 2005, 250, $\mathrm{n}^{\circ} 740$ (datation $c a$. 167-146 av. J.-C. dans la « Maison de Dionysos »). Pour d'autres attestations à Carthage et à Athènes, voir Grace 1934, 277, n 222 et Grace 1956, 145-146, 151 et pl. $65, \mathrm{n}^{\circ} 113$.

37 Blinkenberg 1941, 51-56, spécialement col. 56, nº 89 pour le sculpteur termessien Фí $\lambda \omega v$.

38 Cf. Robert 1966a, 11-13; Foucart 1969, 660 ; Boyxen 2018, 405, tab. 10. L'غ̇ंı $\delta \alpha \mu i ́ \alpha$ était un privilège rhodien régulièrement intermédiaire entre le statut de métèque et celui de citoyen de plein droit. 
ainsi qu'à son épouse $\Theta \varepsilon \sigma \sigma \alpha \lambda i ́ \alpha$, Éphésienne, sans que nous en connaissions le motif.

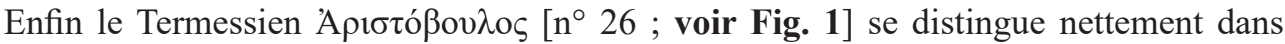
le domaine des liturgies par l'exercice exceptionnel de trois chorégies à Rhodos au $\mathrm{II}^{\mathrm{e}}$

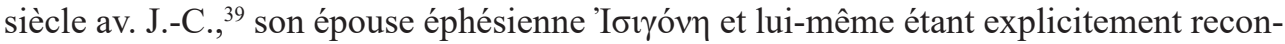
nus comme évergètes. Nous ne connaissons pas l'origine de la fortune de ces membres de l'élite socio-économique rhodienne venus de Pisidie ; s'agissait-il de marchands de vin ? de blé ? d'esclaves?

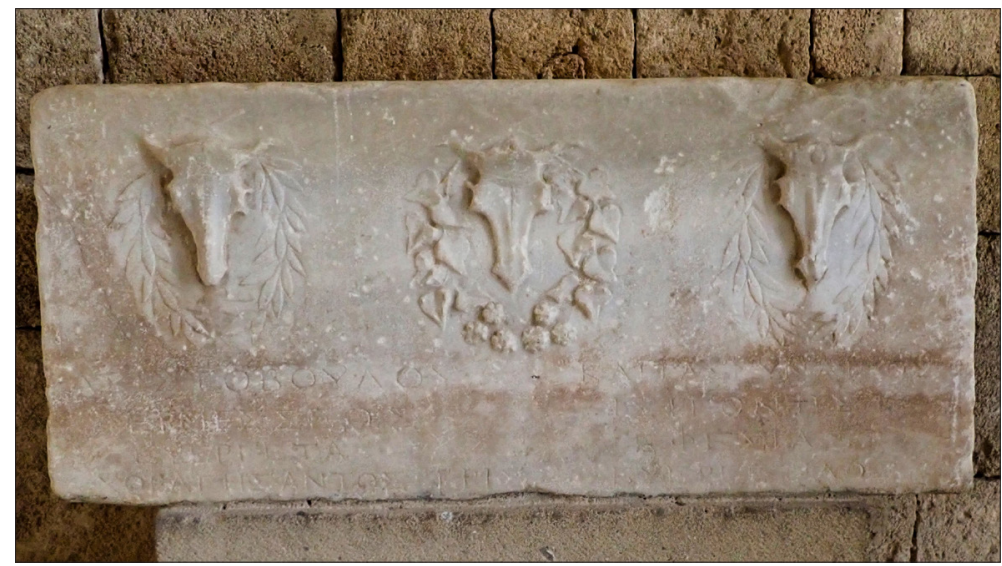

Fig. 1.

Les mercenaires pisidiens n'apparaissent pas explicitement dans les inscriptions dépouillées, mais on peut être sûr qu'il s'en trouve parmi les 53 hommes répertoriés. En effet, au sein une brève synthèse consacrée aux mercenaires pisidiens, M. Launey a souligné que « Comme pour les Lyciens et Pamphyliens, le III siècle [av. J.-C.] semble élargir l'horizon des émigrants pisidiens $»{ }^{40}$ dans la mesure où ces derniers se sont largement engagés dans les rangs des armées lagides, notamment à partir de la troisième guerre de Syrie (246-241 av. J.-C.) et plus généralement entre 246 et 219 pour ce qui concerne la liste de Ras Ibn Hani, ${ }^{41}$ trace documentaire remontant à l'occupation de la côte septentrionale de la Syrie par l'Égypte. Durant le $\mathrm{II}^{\mathrm{e}}$ siècle av. J.-C, probablement entre les années 160 et 140, les mercenaires pisidiens, notamment ceux venus de la petite cité

39 L'attribution de la chorégie semble avoir été régulière pour les étrangers à Rhodes, à l'exemple de Tharsagoras fils de Sôsipatros, trésorier sacré et chorège à Lindos (IG XII, 1, 838); d'Epigonos de Rhodiopolis, métèque affranchi qui fut deux fois chorège à Rhodos (IG XII, 1, 383) ; voir également Jacopi 1932, $193, \mathrm{n}^{\circ} 21$ pour un triérarque, trésorier, prytane et 4 fois chorège ; Maiuri 1925, nº 148 pour un Antiochéen chorège.

40 Launey $1987^{2}$, I, 472. On trouve ainsi des Pisidiens sur des listes de mercenaires à Athènes au début du III ${ }^{\mathrm{e}}$ s. av. J.-C. (IG ${ }^{2}$ II, 1957, l. 17), puis à Tralles dans la seconde moitié de ce même siècle (I. Tralles, 33 ; Robert 1936, 95, l. 10).

${ }_{41}$ Rey-Coquais 1976, 51-61 ; Rey-Coquais 1978, 313-325, avec deux mercenaires d'Etenna, un anonyme (ligne 2) et Apollonios (ligne 26), ainsi qu’un autre Pisidien nommé Hérakleitos (ligne 16). 
reculée d'Adada, sont attestés par une inscription découverte à Amathonte, à Chypre, ${ }^{42}$ possession lagide. On retrouve vers la même époque d'autres Adadéens à Sidon en Phénicie, manifestement aussi au service des Lagides d'après N. Sekunda. ${ }^{43}$ Quant aux Pisi-

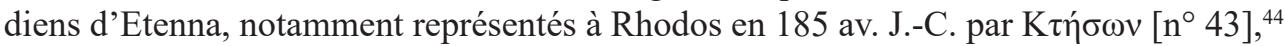
on notera qu'en 218 av. J.-C., leur cité semble avoir fourni d'après Polybe l'effectif massif de 8000 hoplites commandés par Garsyéris (stratège d'Achaios) en vue de défendre la cité pisidienne secondaire de Pednelissos contre la puissante Selge. ${ }^{45}$ À Rhodos, le

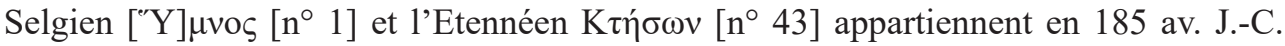
à une association de soldats / mercenaires, comme le montre l'importante inscription de la nécropole orientale de la cité (à Korakonero) publiée en $1989 ;^{46}$ on remarque que ce texte, notamment à verser au dossier de l'association des Panathénaïstes bien connue sur l'île, ${ }^{47}$ date seulement de trois années après le traité d'Apamée, conclusion de la « guerre antiochique » : d'une part on peut donc émettre l'hypothèse que les soldats / mercenaires cités par cette inscription ont pu y participer dans le camp de Rhodes, Pergame et Rome, d'autre part on se souvient qu'Antiochos III fut mis en échec lors de sa tentative du siège de Selge au printemps 193 avant notre ère. ${ }^{48}$

En raison d'une documentation épigraphique lacunaire et souvent peu explicite, c'est l'approche de Rhodes et de la Pisidie dans le contexte historique, géographique et géopolitique régional de la Méditerranée orientale aux époques hellénistique et romaine qui permet de mieux cerner les causes de la présence des Pisidiens sur la grande île du Dodécanèse. Afin de mieux expliquer l'émigration pisidienne telle que M. Launey la décrit brièvement supra, il est essentiel de corréler la pression septentrionale croissante de la colonisation macédonienne (séleucide puis attalide) en Phrygie-Pisidie aux III $^{\mathrm{e}}-\mathrm{II}^{\mathrm{e}}$ siècles avant notre ère ${ }^{49}$ avec l'expansion des Pisidiens de leur région en direction du Sud, jusqu'à la Pamphylie et la Lycie, et de l'Ouest du Taurus, jusqu'à la Carie et au plateau de Tabai. ${ }^{50}$ De la Carie orientale intérieure, les Pisidiens pouvaient rejoindre la Pérée rhodienne, même si leur principal accès à Rhodes dut s'opérer par voie maritime, notam-

42 Stèle funéraire du musée de Limassol (Lemesos) en grès assez dégradé, inv. $\mathrm{n}^{\circ}$ 562/1 [LM 562], découverte dans la région d'Amathonte; vue en octobre 2017. Voir Nicolaou 1976, 366 et fig. 24; RDAC 1975, 152, n 1 ; SEG 26, 1462 ; Robert 1977, 434-435, n 553. Les deux Adadéens sont Bianôr fils de Konôn et Komôn fils de Trokondas.

43 Il s'agit des fameuses tombes peintes de Sidon, aujourd'hui au musée archéologique d'Istanbul. Voir Macridy 1904, 549-556 et pl. XII, stèle conservée à Istanbul montrant par exemple la présence d'un certain $\Sigma \alpha \lambda \mu \alpha \varsigma$ (Zgusta 1964, 451, § 1360-1), originaire d'Adada comme l'indique clairement l'ethnique du texte. Cf. aussi Perdrizet 1904, 234-244, spécialement 239-244 ; Robert 1935, 428-430 ; Sekunda 2006, 68-71, $135-149$.

44 Voir également l'anonyme d'Etenna (non daté) n 45 (à Rhodos).

45 Polybe V.73.3.

46 Kontorini 1989, 73-85, n 10, face B, col. I, l. 22 et col. II, l. 42 ; SEG 39, 737B.

47 Voir Hiller von Gaertringen 1931, 832-834; Blinkenberg 1941, 796 et suiv. ; Pugliese-Carratelli 1939-1940, 147-200 ; Launey 1987², II, 1004-1005, 1014, 1018-1022.

48 Polybe V.73.8 ; V.74.3 ; Tite-Live XXXV.13.5; Grainger 2002, 145-146, 153.

49 Bru 2017, 31-74, 287-289.

50 Voir Strabon XIII.4.13 ; Robert 1954 ; Doni 2009, 213-227. Strabon (XII.7.3) ajoute par ailleurs que des Lélèges s'étaient mêlés aux Pisidiens à de hautes époques. 
ment par le très actif port pamphylien de Sidè, débouché littoral naturel de la Pisidie, ${ }^{51}$ ou encore par Pergè.$^{52}$ Rhodes était, on le sait, une plaque tournante du commerce en Méditerranée orientale très proche des Lagides, aussi faut-il rappeler à titre géopolitique l'entente entre l'île et Ptolémée ${ }^{\text {er }}{ }^{53}$ laquelle a conduit à une coopération commerciale régulière, en dépit d'une brouille passagère avec Ptolémée II lors de la deuxième guerre de Syrie. ${ }^{54}$ Les ports de Pamphylie, où s'exerçait l'influence lagide, ont donc pu facilement livrer des esclaves pisidiens à Rhodes, alors que des mercenaires pisidiens en transit ont pu être régulièrement levés sur l'île par les Lagides. En 219 av. J.-C., Rhodes, Byzance et Cyzique ont servi d'intermédiaires entre Antiochos III et Ptolémée IV lors de la $\mathrm{IV}^{\mathrm{e}}$ guerre de Syrie, ${ }^{55}$ mais c'est en 201 qu'un basculement géopolitique décisif est advenu en Méditerranée orientale, lorsque les Rhodiens et les Pergaméniens firent appel à Rome face à Philippe $\mathrm{V}$ de Macédoine. ${ }^{56} \mathrm{Ce}$ positionnement politique a conduit les Rhodiens à entrer dans l'alliance victorieuse avec Rome et Pergame qui a abouti à la défaite d'Antiochos III en 190-189, au recul géopolitique des Séleucides en Asie Mineure et à l'acmé de Rhodes en vertu du traité d'Apamée de Phrygie de $188:{ }^{57}$ c'est ce qui permit en effet aux Rhodiens d'obtenir de Rome un pouvoir étendu sur la Lycie et sur la Carie jusqu'au Méandre ${ }^{58}$ jusqu'à $167 .{ }^{59}$ De ce fait, la Pisidie devenait une région directement riveraine des Rhodiens, et c'est ce tournant géopolitique régional qui explique manifestement une présence accrue des Pisidiens sur l'île. L'élément venant à mon sens renforcer cette hypothèse est la présence remarquable de 6 Oroandeis à Rhodes $\left[\mathrm{n}^{\circ} 37-\right.$ 42], dont 2 à Rhodos dès le $\mathrm{II}^{\mathrm{e}}$ siècle avant notre ère, Xó $\rho \mu \omega v$ [n $\left.{ }^{\circ} 37\right]$ et un anonyme [ $\left.\mathrm{n}^{\circ} 38\right]$ : probablement convient-il de mettre leur présence en rapport avec l'expédition de Cn. Manlius Vulso qui soumit leur peuple en 189 av. J.-C., ${ }^{60}$ cet acte ayant même suscité de véhémentes critiques de la part des Romains eux-mêmes, en raison d'une violence injustifiée exercée à l'égard d'une population jugée pacifique. ${ }^{61}$ Des esclaves issus du peuple des Oroandeis ont donc pu parvenir à Rhodes après ces événements, mais aussi un peu plus tard à l'occasion des guerres galatiques de 168-166 av. J.-C. survenues entre les Attalides et les Celtes d'Asie ${ }^{62}$ : le fait qu'à cette occasion, lors d'événements

51 Les ressortissants de Sidè sont bien attestés à Rhodes, à l'image de Polyeuktos de Sidè dans la seconde moitié du III ${ }^{\mathrm{e}}$ s. av. J.-C. (belle stèle de la nécropole de Rhodos [inv. n E6547 ] au Musée archéologique de Rhodes, voir Fraser 1977, 10 et 91-92, n. 35 ; cf. aussi Konstantinopoulos 1965, 597, n² 2), ou du sculpteur Thoas de Sidè à Lindos vers 170 av. J.-C. (Blinkenberg 1941, nº 189 et col. 54, nº 65); Sacco 1980, 526 ; pour les 5 personnes de Sidè à Rhodes, dont les deux précitées, cf. notamment Boyxen 2018, 386.

52 À Rhodes, on connaît par exemple la Pergéenne Eủvík

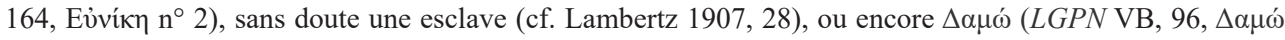
$\left.\mathrm{n}^{\circ} 2\right)$ au $\mathrm{I}^{\mathrm{er}}$ s. avant notre ère ; cf. aussi Morelli 1956, 65 ; Sacco 1980, 525.

53 Diodore XX.81 et 103.

54 Chronique de Lindos 37 ; Polyen V.18 ; Frontin III.9.10 ; Syll. ${ }^{3} 455$.

55 Polybe V.63.5-6.

56 Will 2003², II, 128-129.

57 Polybe XXI.45.1 ; Tite-Live XXXVII.55.7. Voir Holleaux 1931, 304-319 et Holleaux 1932, 7-31.

58 Polybe XXI.45.8; Tite-Live XXXVIII.39.13.

59 Will 2003², II, 298-299.

60 Polybe XXI.41.7 et 43.1 ; Tite-Live XXXVIII.18.2.

${ }^{61}$ Tite-Live XXXVIII.45.9. Sur les Oroandeis, leur territoire et leur soumission à Rome, voir Bru 2017, 172-182, spécialement 173-175.

62 Voir Polybe XXX.1.1-2; XXX.3.2 ; Sartre 2004², 203-204. 
aussi nommés « guerres pisidiques », les cités pisidiennes de Selge ${ }^{63}$ et Amlada ${ }^{64}$ aient soutenu les Galates, sans doute tout comme les cités voisines de Mistea et Vasada qui envoyèrent ensuite des ambassadeurs aux Attalides afin de solder le conflit, ${ }^{65}$ indique que les Pergaméniens durent vendre en esclavage un bon nombre de Pisidiens impliqués en tant que ressortissants des cités évoquées, situées dans le Nord-Est de la Pisidie. Parce que Pergame détint suite au traité d'Apamée le pouvoir sur d'immenses territoires de la moitié occidentale de l'Anatolie entre 188 et 133 av. J.-C. jusqu'à la Pamphylie, il semble assez clair que des esclaves pisidiens furent exportés durant cette période par les Attalides secondés de marchands à partir de cette dernière région littorale, pour certains à Rhodes.

C'est plus tard la première guerre mithridatique qui confirma au I ${ }^{\text {er }} \mathrm{s}$. av. J.-C. les contacts déjà anciens entre les Pisidiens et Rhodes. En 88 av. J.-C., le roi du Pont Mithridate VI Eupator parvint à soulever de nombreuses cités grecques d'Anatolie contre l'autorité de Rome en s'emparant de la Bithynie, de la Phrygie, de la Lycie, de la Pamphylie et de territoires s'étendant jusqu'à l'Ionie. ${ }^{66}$ Face à cette déferlante, un certain Mancinus, ${ }^{67}$ membre de la commission sénatoriale de Manius Aquillius, mais également L. Cassius, proconsul de la province romaine d'Asie créée en 129 , se retirèrent à Rhodes, ${ }^{68}$ laquelle fut attaquée puis assiégée par Mithridate et sa flotte, mais finalement de manière infructueuse ${ }^{69} \mathrm{C}$ 'est de cette époque environ que N. Badoud date l'inscription lacunaire copiée par le médecin et explorateur suédois Johan Hedenborg (1786-1865) affichant en un grand lettrage marqué, à la première ligne conservée, [T]EPMH $\Sigma \Sigma E[---] ;{ }^{70} 1$ 'éditeur de la belle copie d'Hedenborg pense que certains Termessiens pro-romains ont fui à Rhodes, où ils auraient été recrutés afin d'apporter leur aide militaire à Stratonicée de Carie (ligne 2), laquelle fut in fine prise par l'armée de Mithridate, ${ }^{71}$ cet épisode étant toutefois commémoré par l'inscription rhodienne. Plusieurs remarques s'imposent. Il apparaît tout d'abord que la cité de Termessos, même si tous ses habitants ne furent peut-être pas d'accord, se trouva pleinement dans l'alliance romaine en 68 av. J.-C. ${ }^{72}$ L'inscription rhodienne évoquée ressemble à un décret honorifique, peut-être plutôt à la faveur de la communauté des Termessiens (très présents sur l'île), donc au pluriel, au regard du lettrage majeur de la première ligne bien restitué par la copie d'Hedenborg, raison pour laquelle, dans le doute, je n'ai pas créé un anonyme termessien dans le catalogue des Pisidiens de Rhodes donné en annexe. Il y a plus : alors que l'ethnique des Termessiens de Rhodes [ $\left.{ }^{\circ} 26,28-34\right]$ est toujours orthographié avec un epsilon (E) en deuxième syllabe, on remarque dans l'inscription d'Hedenborg que l'ethnique en question, qu'il soit au singulier pour un Termessien remarquable, voire héroïque, ou au pluriel, est orthographié avec un èta $(\mathrm{H})$ en deuxième syllabe. Or la seule autre même

${ }^{63}$ Polybe XXXI.1.1-2; Trogue Pompée, Prologue, 34.

${ }_{64}$ Qui fut sanctionnée par les Attalides, cf. Welles $1966^{2}, 237-241, \mathrm{n}^{\circ} 54$.

${ }_{65}$ Swoboda - Keil - Knoll 1935, n 74 ; cf. Flacelière - Roussel 1936, 386.

${ }^{66}$ Appien, Mithr. 76-77.

67 Peut-être T. Manlius Mancinus, tribun en 108-107 av. J.-C. d'après Goukowski 2003, 146-147, n. 179.

68 Appien, Mithr. $75 ; 94$.

69 Appien, Mithr. 85 ; 94-106 ; Peyras 2010, 157-174.

70 Badoud 2017, 25-30, n 2 et pl. 29, n 263.

71 Appien, Mithr. 82.

72 ILS 38 ; Sherk 1984, 89-92, n 72 ; Ferrary 1985, 419-457. 
exception se trouve dans la liste de noms (avec ethniques) où figure le Termessien $[\mathrm{O} \tau]$

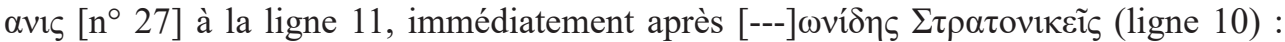
cette liste ressemblant tout à fait à un inventaire de mercenaires d'origines diverses et parfois lointaines (d'Athènes à Séleucie du Tigre, en passant par Damas) jusqu'ici datée des II $^{\mathrm{e}}-\mathrm{I}^{\mathrm{er}}$ s. av. J.-C. (du I ${ }^{\mathrm{er}}$ s. av. J.-C. d'après L. Robert) ${ }^{73}$ est à rapprocher de l'inscription d'Hedenborg, peut-être vers 88 av. J.-C., sachant que dans les deux cas le graveur ne semble pas suivre la graphie rhodienne habituelle de l'ethnique des Termessiens. À Rhodes, les Termessiens sont présents au moins depuis le $\mathrm{II}^{\mathrm{e}}$ siècle (à Lindos avec

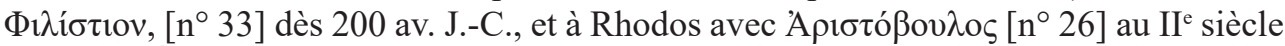
av. J.-C.). À propos de ce qui précède, on notera au passage que le seul anthroponyme de nos Pisidien(ne)s de Rhodes qui ne soit ni hellénique ni anatolien, mais iranien, est celui

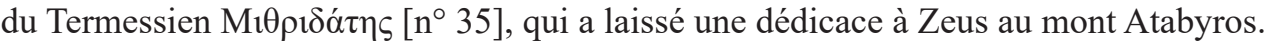

En 63 av. J.-C., un discours de Cicéron nous informe que le consul romain de 79 avant notre ère P. Servilius Vatia Isauricus soumit par son expédition les Oroandeis entre 78 et 74 av. J.-C., leurs territoires étant incorporés à l'ager publicus, ${ }^{74}$ ce qui constituait pour Rome un moyen de compléter sur les marges orientales de la province d'Asie les territoires acquis lors de la paix de Dardanos de 85 av. J.-C. traitée avec Mithridate VI Eupator. À cette occasion, de nouveaux esclaves pisidiens purent être exportés, notamment à Rhodes. Puis, peu avant sa mort en 25 av. J.-C., le roi de Galatie Amyntas, client de Rome, entreprit une campagne militaire assez dure en Phrygie-Pisidie, ${ }^{75}$ où il fut tué par ses ennemis louvitophones nommés Homonadenses dans les sources latines, parmi lesquels se trouvaient notamment des Pisidiens et des Isauriens, peuples voisins dont les survivants capturés furent sans surprise vendus en esclavage. Suite à la création de la province romaine de Galatie et d'un réseau de colonies au Sud du Taurus (Antioche de Pisidie, Kremna et Lystra par exemple) par Auguste, la soumission de la Pisidie fut brutalement parachevée au nom de Rome par P. Sulpicius Quirinius, gouverneur de Galatie vers 5-3 av. J.-C., ${ }^{76}$ date très probable de la campagne contre les Homonadenses (4-3 av. J.-C. d'après R. Syme), ${ }^{77}$ lesquels pillaient la région à partir de bases situées aux confins de la Pisidie et de l'Isaurie ${ }^{78}$ entre la ville moderne de Seydişehir et le lac Trogitis (Suğla). La violence d'État telle qu'elle fut pratiquée par Rome, y compris aux yeux de Strabon, implique que de nombreux esclaves pisidiens furent mis en vente sur les marchés vers cette époque, dont sans aucun doute à Rhodes.

En conclusion, les Pisidien(ne)s recensé(e)s à Rhodes et dans sa Pérée carienne confirment d'abord la puissance et l'influence de Selge, ${ }^{79}$ dont l'hellénisation fut précoce, ensuite l'activité et la coopération dont Termessos la Grande était capable. Sur ce dernier point, on pourrait par exemple citer la convention d'alliance que Termessos contracta

73 La graphie du texte confirme effectivement une telle datation.

74 Cicéron, De lege agraria II.50. Sur l'itinéraire de la campagne d'Isauricus et la seconde soumission des Oroandeis à Rome (après l'expédition de Cn. Manlius Vulso en 189 av. J.-C.), cf. Syme 1995, 211-212.

75 Strabon XII.6.4.

76 PIR ${ }^{2}$ S 1018 ; Dąbrowa 1998, 27-30 et 139, n. 159-160.

77 Voir Syme 1995, 257-269; Levick 1967, 203-214.

78 Voir Strabon XII.6.5.

79 Bru 2017, 4, 29, 52, 223, 250-251, 254. 
avec la plus modeste cité d'Adada au II ${ }^{\mathrm{e}}$ s. av. J.-C., ${ }^{80}$ gage de bonnes relations diplomatiques entre elles qui ont éventuellement pu faciliter des contacts entre la Pisidie centrale et Rhodes, sachant que Termessos se rangea finalement, certes plus tard que l'île du Dodécanèse, aux côtés des Romains. Les Oroandeis, ayant pour leur part subi de plein fouet l'hégémonie de Rome, se sont soumis totalement à cette dernière, alors que la cité d'Etenna confirme par ses ressortissants attestés à Rhodes son statut de « réservoir » de soldats / mercenaires constaté ailleurs. Retenons que ce sont d'abord les cités du Sud de la Pisidie (Selge, Termessos, Etenna, Erymna et Sagalassos) qui fournirent des candidats (volontaires ou involontaires) à l'émigration vers Rhodes et sa Pérée, d'une part en raison de leur proximité avec la plaine pamphylienne et ses ports, d'autre part probablement en raison de leur degré d'hellénisation.

On se doit d'évoquer celles et ceux qui se disent sans précision « Pisidien(ne)s » dans les inscriptions rhodiennes, selon plusieurs hypothèses. S'ils ne se rattachent par leur ethnique à aucune polis particulière de Pisidie, on pourrait en conclure qu'ils se situent « hors-cités », préférant mentionner un ethnique plus général mais réputé (les Pisidiens s'étant au fil de l'antiquité gréco-romaine fait un nom dans le domaine militaire), plutôt que de mentionner une communauté louvitophone obscure. Pour des motifs analogues et peut-être identitaires, certains Pisidiens issus d'une cité ont pu préférer une appellation plus générale, dans le sens latin de natio, qui correspond à un groupe culturel plus large. L'autre éventualité serait que ces Pisidiens aient vécu à Rhodes depuis plusieurs générations, particulièrement dans le milieu servile, sans se rattacher désormais à une cité originelle particulière, mais il semble que dans ce cas les habitudes rhodiennes

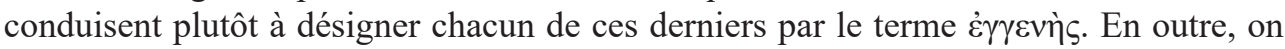
pourrait s'étonner de l'absence de la mention de ressortissants provenant de cités telles que Kremna, Adada, Tymbriada ou Pednelissos ; le fait est que ces cités de Pisidie se trouvaient dans des régions relativement reculées (surtout Adada et Tymbriada) qui ont éventuellement pu les exclure ou les protéger plus longtemps des contacts avec les puissances géopolitiques régionales recherchant mercenaires et esclaves.

Parmi les 61 Pisidien(ne)s recensé(e)s, outre les esclaves supposé(e)s (qui bénéficièrent cependant d'un monument funéraire), une relative intégration sociale peut être constatée à Rhodes et dans sa Pérée, dans la mesure où quelques notables et évergètes se dégagent de l'ensemble, dans un contexte politique et socio-économique qui paraît s'être montré assez accueillant au regard des standards antiques, ${ }^{81}$ peut-être encore davantage aux $\mathrm{II}^{\mathrm{e}-\mathrm{I}^{\mathrm{er}}}$ s. av. J.-C., après l'année 167 av. J.-C. qui vit l'île du Dodécanèse relativement décliner sur le plan économique en raison des Romains, lesquels imposèrent d'une part la création du port franc concurrent de Délos, d'autre part une rétrocession des pouvoirs de Rhodes sur sa Pérée. Cette intégration sociale, ou socioéconomique, connut des limites : ainsi, pour 61 personnes, seulement deux semblent

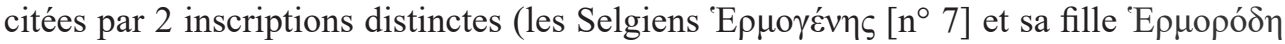
$\left[n^{\circ} 8\right]$ ) ; peut-être pourrait-on légèrement améliorer cette statistique si certains autres

80 TAM III, 1, 2.

81 Voir Strabon XIV.2.5 ; Migeotte 2014, 291-292. Une loi ancestrale et les hauts revenus commerciaux de Rhodes permirent une aide au « petit peuple » de l'île en échange de travaux consentis pour la construction et l'entretien de la flotte, par un «pacte social » dont les premiers bénéficiaires furent nécessairement les citoyens de plein droit. 
textes rhodiens nous fournissaient ethniques et patronymes. Force est de constater que l'immense majorité des textes cités en référence dans notre catalogue sont de courtes et modestes épitaphes. Sauf pour une famille (nos $\left.n^{\circ} 7-8\right)$ dont nous connaissons partiellement la parenté, les groupes familiaux n'apparaissent pas en dehors des couples (mariés ou non), en l'absence d'enfants ou de parents mentionnés. Une endogamie anatolienne apparaît en revanche très clairement pour les Pisidien(ne)s de Rhodes, marié(e)s ou non :

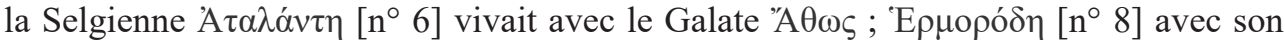

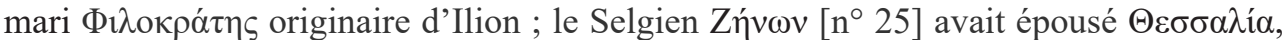

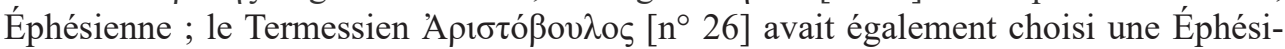

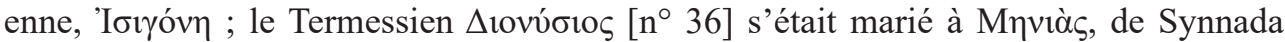

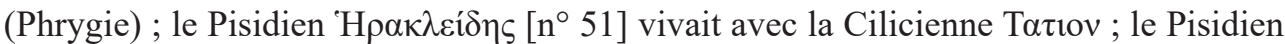

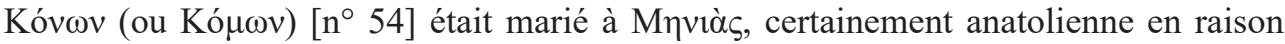
de son anthroponyme théophore se rapportant au dieu Mên ; les seules exceptions sont

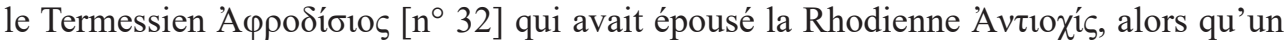

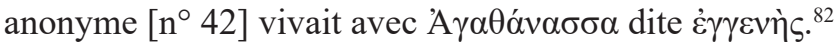

Présents à Rhodes au moins à partir du $\mathrm{III}^{\mathrm{e}} \mathrm{s}$ av. J.-C., surtout aux $\mathrm{II}^{\mathrm{e}}-\mathrm{I}^{\mathrm{er}}$ s. av. J.-C., et ce jusqu'au I ${ }^{\mathrm{er}} \mathrm{s}$. de notre ère, on y perd ensuite la trace documentaire des Pisidiens, lesquels furent pour l'essentiel intégrés aux provinces romaines de Galatie et de LyciePamphylie.

\section{Catalogue}

Le catalogue qui suit recense les Pisidiens et Pisidiennes par leur lieu d'origine, puis par leur lieu de résidence à Rhodes ou dans sa proche Pérée carienne; chaque notice précise l'anthroponyme, l'ethnique, la nature des documents utilisés, le lieu exact de découverte et une datation lorsque cela est possible, puis les références épigraphiques (ou archéologiques), bibliographiques et onomastiques essentielles.

\section{SELGE}

\section{Rhodos}

1. $\left[{ }^{\circ} \mathrm{Y}\right] \mu \nu \mathrm{\nu} \varsigma-[\Sigma] \varepsilon \lambda \gamma \varepsilon u ́ \varsigma$-liste de souscription associative de la nécropole orientale (Korakonero) -185 av. J.-C. - Kontorini 1989, 73-85, n 10, face B, col. II, l. 42 ; SEG 39, 737B ; I. Selge T48; LGPN VC, 433, "Y $\mu$ vo $\mathrm{n}^{\circ} 3$.

2. Máp $\omega v-\Sigma \varepsilon \lambda \gamma \varepsilon u ́ \varsigma$-fabricant d'amphores connu par timbres amphoriques $-145-108$ av. J.-C. -ASCSA, ABL 64, 30.5.60 ; Centre Alexandrin d'Étude des Amphores inv. $\mathrm{n}^{\circ}$ ALEX ABC 0374.16 ; Badoud 2017, 26, n. 15 ; Boyxen 2018, 409, tab. 14, $\mathrm{n}^{\circ} 13$ et p. 411 , tab. $15, \mathrm{n}^{\circ} 97$.

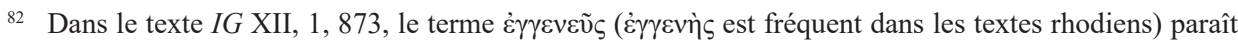
concerner une esclave née sur l'île de Rhodes (voir Bresson 1997, 119). 


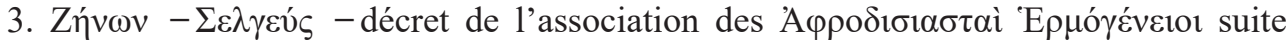
à la proposition du personnage, évergète (stèle de marbre gris découverte en 1919 dans la nécropole orientale ; L. 0.63m; H. 0.60m ; P. 0.16m) -vers $109-108$ av. J.-C. -Maiuri 1921-1922, 223-232 ; SEG 3, 674, ll. 1, 3, 20 ; Pugliese-Carratelli 19391940, 156-165, nº 18 ; Fraser 1977, 61 et 151 n. 346; I. Selge T53 ; Boyxen 2018,

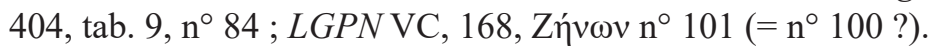

4. 'Oỵó $\sigma \mu \rho_{\varsigma}-\Sigma \varepsilon \lambda \gamma \varepsilon v ́ \varsigma-$ stèle funéraire fragmentaire de marbre bleuâtre découverte dans la ferme d'Anastasios Dimitriadis proche de la ville (L. $0.26 \mathrm{~m} ;$ H. $0.27 \mathrm{~m} ;$ P. $0.08 \mathrm{~m}$ )

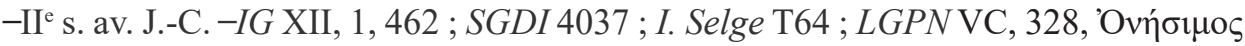
$\mathrm{n}^{\circ} 157$.

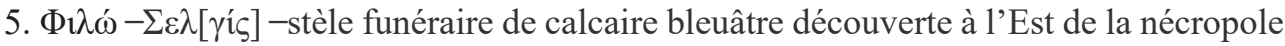
orientale (Korakonero ; L. $0.235 \mathrm{~m}$; H. $0.34 \mathrm{~m}$; P. $0.08 \mathrm{~m}$ ) - II ${ }^{\mathrm{e}}$ s. av. J.-C. -Kontorini

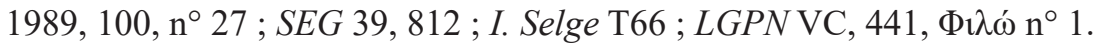

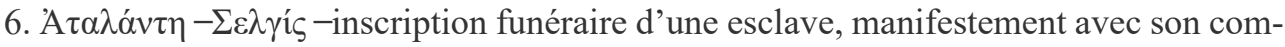
pagnon 'A $\theta \omega \varsigma$, Galate (autel funéraire orné de bucranes et guirlandes, découvert dans

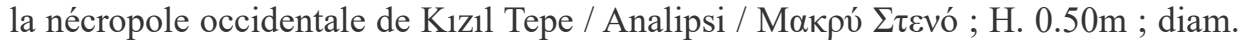
$0.35 \mathrm{~m})-\mathrm{II}^{\mathrm{e}}-\mathrm{I}^{\text {er }}$ s. av. J.-C. -Foucart 1866b, 332, $\mathrm{n}^{\circ} 53$; Biliotti - Cottret 1881, 493 ; IG XII, 1, 548 ; I. Selge T58 ; Fraser 1977, 56 et 144 n. 306 ; LGPN VC, 72,

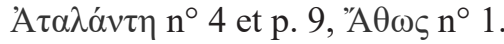

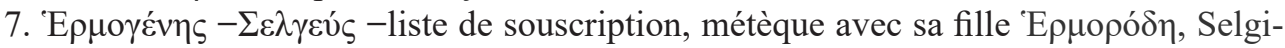
enne (imposante plaque de marbre L. $1.30 \mathrm{~m}$; H. $1.12 \mathrm{~m}$; P. $0.30 \mathrm{~m}$ ) ; inscription funéraire $-\mathrm{I}^{\mathrm{er}}$ s. av. J.-C. -Jacopi 1932, 177-179, nº 6, col. II, l. 27 ; Foucart 1889, 367, n 4 =IG XII, 1, $461=$ SGDI 4036 ; Maiuri 1925, 93, $\mathrm{n}^{\circ} 188=I$. Selge T62 (urne cinéraire fragmentaire en marbre découverte entre Rhodos et Asgourou) ; Robert 1966a, 12 ;

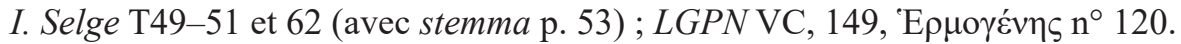

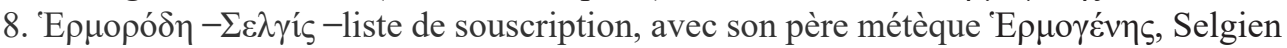
(imposante plaque de marbre L. $1.30 \mathrm{~m}$; H. $1.12 \mathrm{~m}$; P. $0.30 \mathrm{~m}$ ) ; inscription funéraire

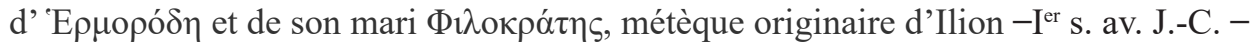
Jacopi 1932, 177-179, $\mathrm{n}^{\circ}$ 6, col. II, ll. 29 et 34 ; Foucart 1889, 363-366 = IG XII, 1, 157, ll. 15-16 = SGDI 3838 ; Robert 1966a, 12 ; I. Selge T49-50 ; Vidmann 1969,

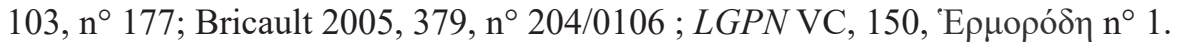

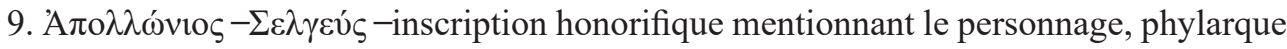

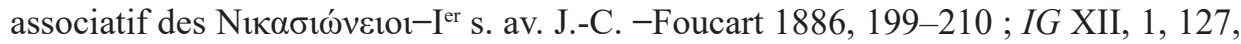
face A, col. B, l. 35 ; Hiller von Gaertringen 1896, 60-61 ; SGDI 4108 ; I. Selge T54 ; $L G P N$ VC, 45, А $\mathrm{Ao \lambda \lambda \omega ́vios} \mathrm{n} 578$.

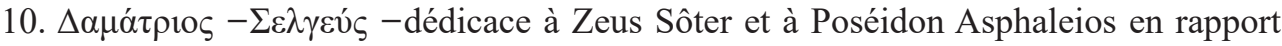
avec l'association des $\Sigma \omega \tau \eta \rho 1 \alpha \sigma \tau \alpha i ́-e ́ p o q u e ~ h e l l e ́ n i s t i q u e-K o n s t a n t i n o p o u l o s ~ 1968$,

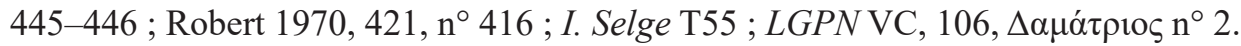

11. 'A $\theta \alpha v \alpha[---]-\Sigma \varepsilon \lambda \gamma \varepsilon[\hat{\zeta} \varsigma]-$ inscription funéraire découverte en remploi dans un mur du quartier turc de Rhodes -époque hellénistique-Maiuri 1925, 92, nº 186 ; I. Selge T56.

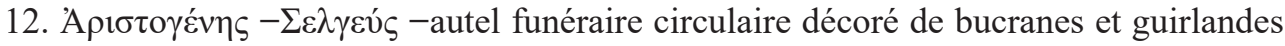
(H. 0.495m) -époque hellénistique -Maiuri 1925, 92, n 187 ; I. Selge T57 ; LGPN

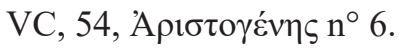




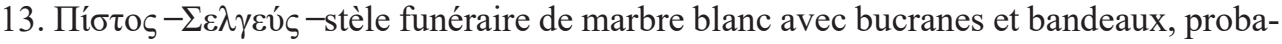
blement pour un esclave -époque hellénistique -Hiller von Gaertringen 1896, 47,

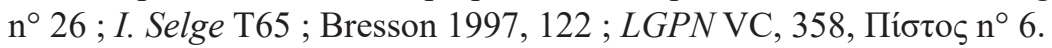

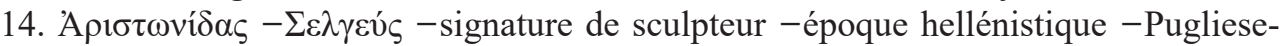

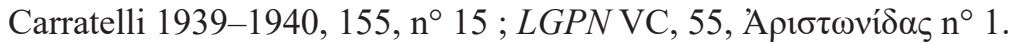

15. $\Delta \alpha \mu \alpha \tau \rho i ́ \alpha-\Sigma \varepsilon \lambda \gamma \grave{s} \varsigma$-petite stèle funéraire de marbre à fronton triangulaire, découverte au village de Zakri (L. 0.20m ; H. 0.50m ; P. 0.075m) -basse époque hellénistique début d'époque impériale -Porro 1916, 131, n 21 ; I. Selge T59; LGPN VC, 106, $\Delta \alpha \mu \alpha \tau \rho i ́ \alpha \mathrm{n}^{\circ} 1$.

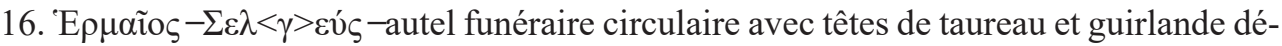

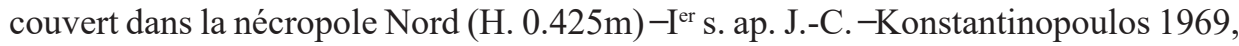

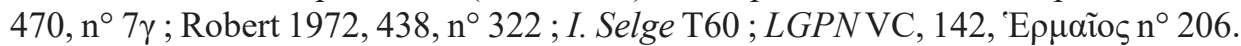

17. Zńvœv $-[\Sigma] \varepsilon \lambda \gamma \varepsilon v ́ \varsigma$-autel funéraire circulaire avec représentation d'un repas funéraire -basse époque hellénistique - début d'époque impériale-Fraser 1977, 32 et 124 n. 171, et fig. 86c ; SEG 27, 472 ; I. Selge T63; LGPN VC, 168, Zńv $\omega v$ n $^{\circ} 102$.

18. [--- $] \sigma[---]-\Sigma \varepsilon \lambda \gamma \varepsilon v ́ \varsigma-$ fragment d'une stèle funéraire de marbre de la nécropole occi-

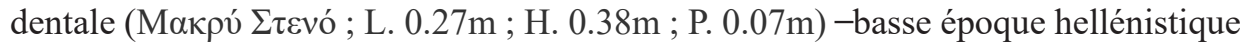
- début d'époque impériale -Maiuri 1925, 93, n $189 ;$ I. Selge T67.

19. $\Sigma$ PA.O $\Sigma-\Sigma \varepsilon \lambda<\gamma>\varepsilon v ́ \varsigma$-autel funéraire circulaire en marbre lartien $(\mathrm{H} .0 .41 \mathrm{~m}$; diam. $0.32 \mathrm{~m})$ découvert dans la nécropole Nord -Konstantinopoulos $1969,470, \mathrm{n}^{\circ} 2 \delta$; Robert 1972, 438, n 322 ; I. Selge T61.

\section{Lindos}

20. [---] $\varsigma-\Sigma \varepsilon \lambda \gamma \varepsilon v ́ \varsigma-$ liste fragmentaire de marbre blanc endommagée comportant anthroponymes et ethniques - fin $\mathrm{II}^{\mathrm{e}} \mathrm{s}$. - début ${ }^{\mathrm{Ier}}$ s. av. J.-C. -Blinkenberg 1941, 582-585, $\mathrm{n}^{\circ} 275$, l. $41 ;$ I. Selge $\mathrm{T} 68$.

21. [---]KI $\Delta \mathrm{A}[---]-\sum \varepsilon \lambda \gamma \varepsilon v ́ \varsigma$-fragment d'une stèle funéraire de marbre sombre ornée d'un kymation lesbien, découvert à Sianna (territoire de Lindos, dèmos des Brasioi) début de l'époque impériale-Konstantinopoulos 1963, 31, nº 63 ; Robert 1966b, 407, $\mathrm{n}^{\circ} 298 ;$ I. Selge T69.

22. [---] $-\Sigma \varepsilon \lambda \gamma \varepsilon v ́[\varsigma]$-fabricant d'amphores connu par timbre amphorique-Nilsson 1909, 62, 67, 103-104, 274, n 371 ; Robert 1963, 378-379, n. 6 ; I. Selge T70 ; Boyxen 2018,409 , tab. $14, n^{\circ} 14$ et p. 412 , tab. $15, n^{\circ} 161$.

\section{Mont Atabyros}

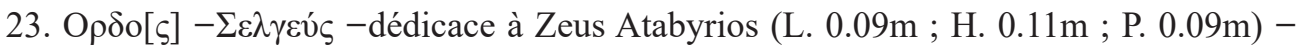
III'-II ${ }^{\mathrm{e}}$ s. av. J.-C. -Jacopi 1932, 241, n 161 ; Robert 1963, 431-432 ; Zgusta 1964,

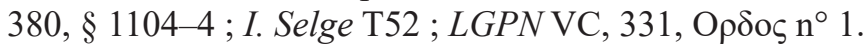




\section{Tymnos (Pérée rhodienne)}

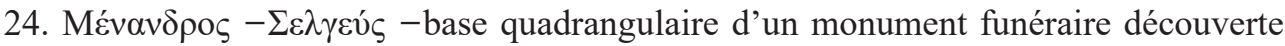
au village d'Avlana (L. $0.51 \mathrm{~m}$; H. $0.34 \mathrm{~m}$; P. $0.43 \mathrm{~m}$ ) -probablement basse époque hellénistique - Fraser-Bean 1954, 42, n 29 ; SEG 14, 706 ; Robert 1955, 265, ${ }^{\circ}$

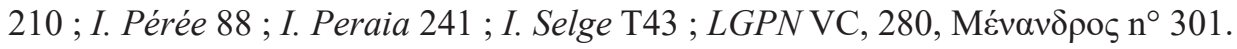

\section{Kasara (Pérée rhodienne)}

25. Zńv $\omega v-\Sigma \varepsilon \lambda \gamma \varepsilon v ́ \varsigma$-dédicace du koinon des Asklapiastes, avec honneurs de deux couronnes au personnage, ainsi qu'à son épouse $\Theta \varepsilon \sigma \sigma \alpha \lambda i ́ \alpha$, Éphésienne - fin $\mathrm{II}^{\mathrm{e}}-$ début $\mathrm{I}^{\mathrm{er}}$ s. av. J.-C.-Hicks 1889, 49-50, n 4 ; Chaviaras 1913, 3, nº 87 ; I. Pérée $169 ;$ I.

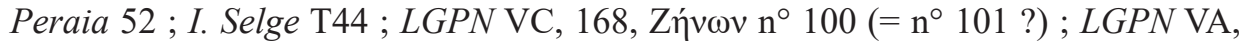
217, $\Theta \varepsilon \sigma \sigma \alpha \lambda i \alpha n^{\circ} 1$.

\section{TERMESSOS}

\section{Rhodos}

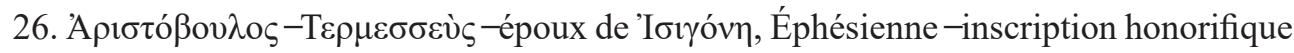
-le personnage fut trois fois chorège, les deux membres du couple furent reconnus comme évergètes $-\mathrm{II}^{\mathrm{e}}$ siècle av. J.-C. -IG XII, 1, 385 ; Fraser 1977, 36 et 130 n. 204 ;

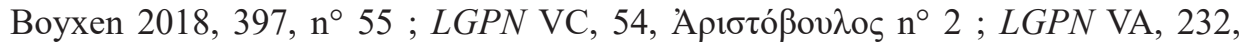

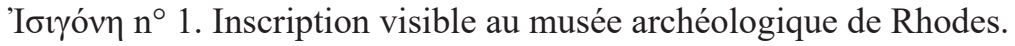

27. [O $\tau] \alpha v i \varsigma-T \varepsilon \rho \mu \eta \sigma \sigma \varepsilon \dot{v} \varsigma$-liste de noms avec ethniques $-\mathrm{II}^{\mathrm{e}}-\mathrm{I}^{\mathrm{er}}$ s. av. J.-C.-PuglieseCarratelli 1952-1954, 287-289, n 63, l. 11 ; Jones 1992, 123-132, spécialement

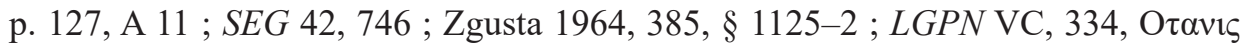
$\mathrm{n}^{\circ} 2$.

28. $\Phi \hat{i} \lambda<\omega>v-T \varepsilon \rho \mu \varepsilon \sigma<\sigma>\varepsilon v ̀ \varsigma$-signature de sculpteur $-100-50$ av. J.-C. -Scrinzi 1899, 19-23, no 11, l. 7 ; IG XII, 1, 108 ; Blinkenberg 1941, 56, nº 89 ; LGPN VC, 441, Tí $_{\omega v} \mathrm{n}^{\circ} 11$; Vollkommer 2001-2004, n ${ }^{\circ} 8$; cf. aussi Foucart 1866a, 161-163.

29. 'А $\rho \varepsilon \mu 1 \varsigma-T \varepsilon \rho \mu \varepsilon \sigma \sigma i ́$, -autel circulaire de marbre à bucranes et guirlandes (h. $0.43 \mathrm{~m}$;

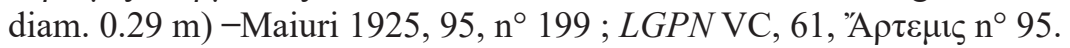

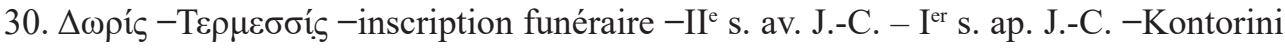
$1975,38, \mathrm{n}^{\circ} 5 ; L G P N \mathrm{VC}, 132, \Delta \omega$ pís $^{\circ}{ }^{\circ} 4$.

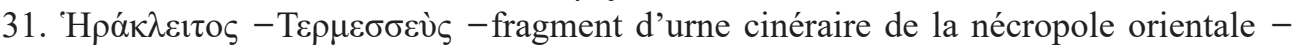

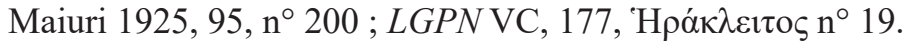

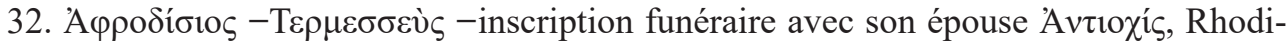
enne-Foucart 1866b, 334, n 55 ; IG XII, 1, 544 ; Fraser 1977, 48 et 138 n. $247 ; 56$

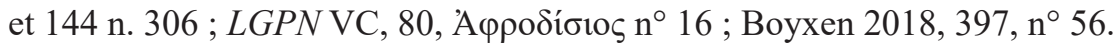




\section{Lindos}

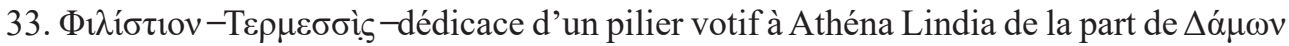

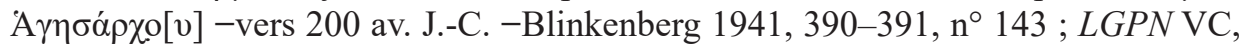

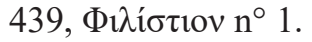

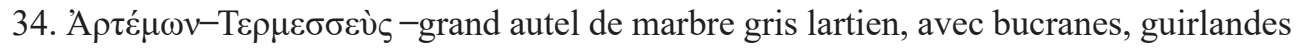
et couronne, découvert à Chochlakas, près de Plymmiri (au Sud-Est de l'île) - ${ }^{\text {er }}$ siècle av. J.-C. -Maiuri 1925, 136, n 431 ; Berges-Nollé 1996, 131, n 130 et pl. 38, 4 ;

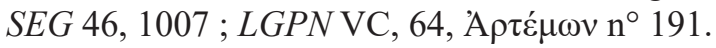

\section{Mont Atabyros}

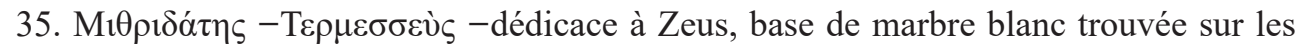
pentes du mont Atabyros -époque impériale -Jacopi 1932, 245, $\mathrm{n}^{\circ} 179$; LGPN VC,

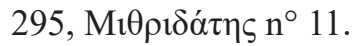

\section{Tymnos (Pérée rhodienne)}

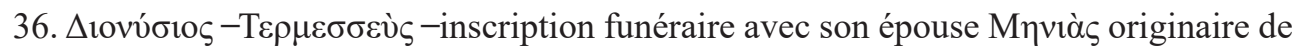
Synnada -II ${ }^{\mathrm{e}}-\mathrm{I}^{\mathrm{er}}$ S. av. J.-C. -Chaviaras 1911, 65, n 62 ; I. Peraia 211 ; I. Pérée 98 ;

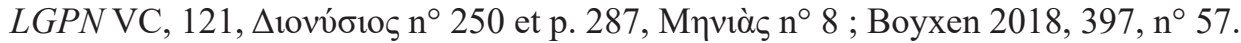

\section{OROANDEIS}

\section{Rhodos}

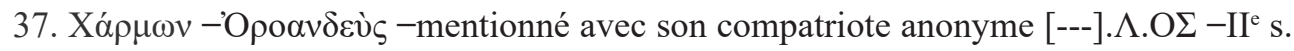
av. J.-C. -IG XII, 1, 448 ; Boyxen 2018, 378 ; LGPN VC, 449, Xáp $\mu \omega v \mathrm{n}^{\circ} 1$.

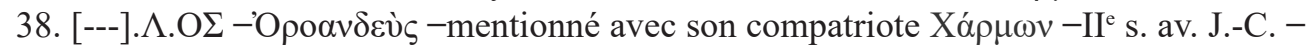
IG XII, 1, 448 ; Boyxen 2018, 378.

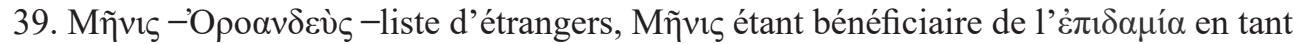
que résident $-\mathrm{I}^{\mathrm{er}}$ s. av. J.-C. -Jacopi 1932, 177, nº 6, col. II, l. 16 ; Boyxen 2018, 378 et $405 ; L G P N$ VC, 289, Mñvis nº 255.

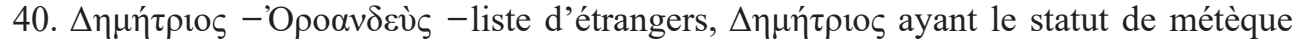

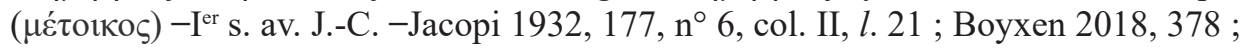
$L G P N$ VC, 111, $\Delta \eta \mu \eta ́ \tau \rho ı \varsigma ~ n^{\circ} 275$.

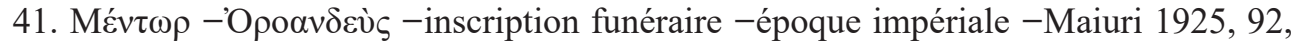

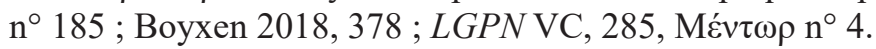




\section{Lindos}

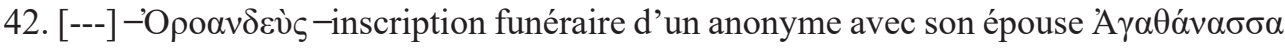

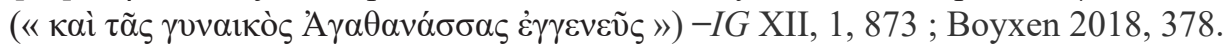

\section{ETENNA}

\section{Rhodos}

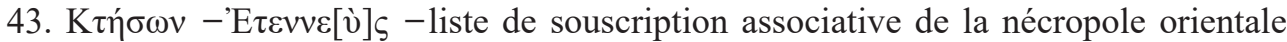
(Korakonero) -185 av. J.-C. -Kontorini 1989, 73-85, $\mathrm{n}^{\circ}$ 10, face B, col. I, l. 22 ; SEG

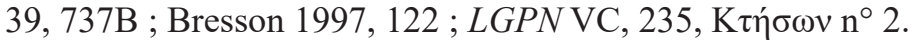

44. [---]-['E] $\tau \varepsilon v v \varepsilon v ̀ \varsigma-$ base rectangulaire d'un monument funéraire (L. 0.34m; H. 0.22m ; P. $0.43 \mathrm{~m}$ ) découverte à Koskinou, près du lieu dit « Tombeau de Ptolémée » (à environ $2 \mathrm{~km}$ au Sud de la ville de Rhodes, à l'Est d'Asgourou)-Hatzfeld 1910, 244, $\mathrm{n}^{\circ} 17$; Sacco 1980, 520 ; Boyxen 2018, 361.

\section{ERYMNA}

\section{Rhodos}

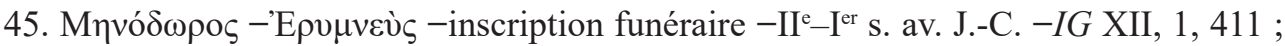
Robert 1963, 378 ; Sacco 1980, 520 ; Zgusta 1984, 449, § 952-1 ; LGPN VC, 291,

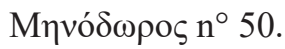

\section{SAGALASSOS}

\section{Rhodos}

46. Eưvovৎ $-\Sigma \alpha \gamma \alpha \lambda \alpha \sigma \sigma \varepsilon v ́ \varsigma$-autel funéraire quadrangulaire de calcaire, orné de bucrane et guirlande (L. $0.55 \mathrm{~m}$; H. $0.24 \mathrm{~m}$; P. $0.55 \mathrm{~m}$ ) découvert dans la nécropole occidentale (Monte Smith) -Ir ${ }^{\text {er }}$ s. av. J.-C. -Porro 1916, 128, n 11 ; Morelli 1956, 167 ; Bresson 1997, $122 ; L G P N$ VC, 157, Eưvouৎ n 6. 


\section{PISIDIE}

\section{Rhodos}

47. Xĩos $-\Pi 1 \sigma i ́ \delta \alpha \varsigma$-liste de souscription associative de la nécropole orientale (Korakonero) -185 av. J.-C. -Kontorini 1989, 73-85, n 10, face B, col. I, l. 26 ; SEG 39, 737B ; Bresson 1997, 122 ; LGPN VC, 449, Xĩos n 2.

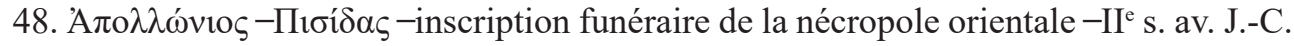

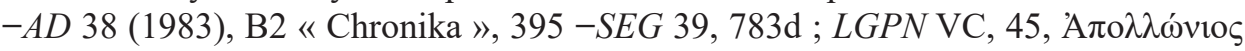
$\mathrm{n}^{\circ} 536$.

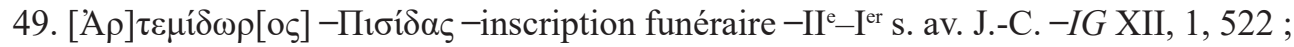

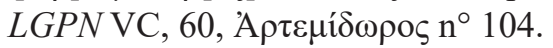

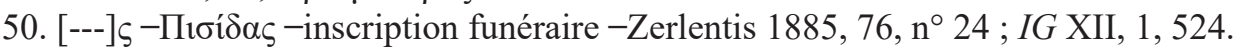

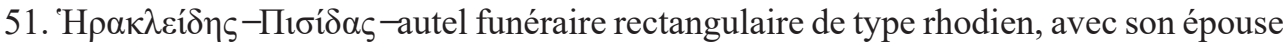

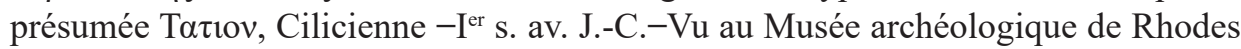
en Mars 2020.

52. [Трок] ov $\delta \alpha \varsigma_{\varsigma}-\Pi 1 \sigma i ́ \delta \alpha_{\varsigma}$-autel funéraire cylindrique en marbre lartien découvert dans la nécropole Nord ; H. 0.36m ; diam. 0.25m -Konstantinopoulos 1969, 470, ${ }^{\circ} 2 \varepsilon$; Zgusta 1964, 490-492, § 1512-31; Robert 1972, 438, n 322 ; Sacco 1980, 525.

53. А $А \tau \varepsilon \mu i ́ \delta \omega \rho о \varsigma-\Pi 1 \sigma i ́ \delta \alpha \varsigma$ - autel funéraire cylindrique en marbre lartien orné de bucranes et guirlandes ; musée archéologique de Rhodes (H. 0.24m ; diam. 0.185m) époque hellénistique-Berges-Nollé 1996, 150 et 160, nº 268 ; SEG 46, 1039 ; LGPN VC, 60, А $А \tau \varepsilon \mu i ́ \delta \omega \rho o \varsigma n^{\circ} 103$.

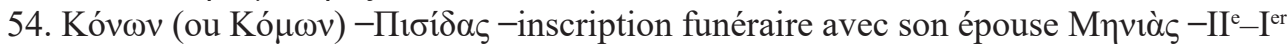

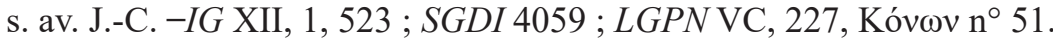

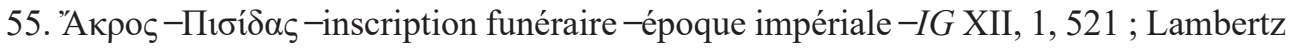

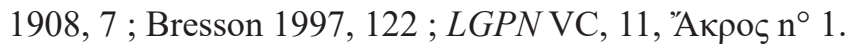

\section{Ialysos}

56. Kavov $-\Pi 1 \sigma i ́ \delta \alpha \varsigma$-inscription funéraire - $\mathrm{II}^{\mathrm{e}}$ s. av. J.-C. $-I G$ XII, 1, 685 ; Lambertz 1908, 17 ; Zgusta 1964, 213, § 523-4 ; Bresson 1997, 122 ; LGPN VC, 209, Kavov $\mathrm{n}^{\circ} 1$.

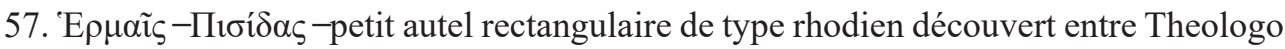
et Soroni -basse époque hellénistique ou début d'époque impériale-Papachristodou-

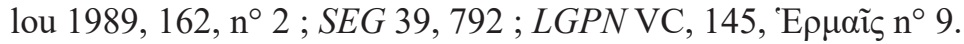

58. Eưvoı $\alpha-\Pi 1 \sigma i ́ \delta ı \sigma \alpha-$ autel funéraire cylindrique (cimetière près de Trianda) ; H. $0.47 \mathrm{~m}$; diam. $0.37 \mathrm{~m}$-époque hellénistique - Marangou - Papachristodoulou 1993, 538 ; SEG 47, 1246 ; LGPN VC, 156, Eưvor $\alpha$ n $^{\circ} 3$. 


\section{Lindos}

59. $\Delta$ í $\sigma \kappa о \varsigma-П 1 \sigma i ́ \delta \alpha \varsigma-i n s c r i p t i o n$ funéraire-III - II ${ }^{\mathrm{e}}$ s. av. J.-C. $-I G$ XII, 1, 879 ; Lambertz

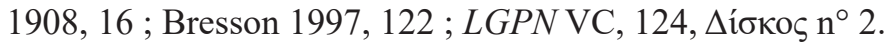

60. $\Sigma \pi \alpha ́ \rho \tau \alpha-\Pi 1 \sigma i ́ \delta ı \sigma \alpha$-inscription funéraire de deux femmes esclaves (l'autre étant

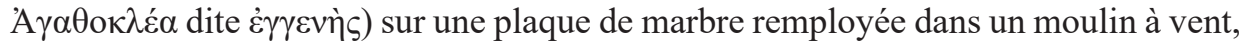
à Asklipio ( $c a .15 \mathrm{~km}$ au Sud-Ouest de Lindos) -probablement $\mathrm{I}^{\text {er }}$ S. av. J.-C. -Heden-

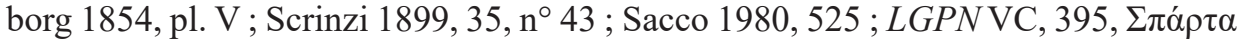
$\mathrm{n}^{\circ} 1$.

\section{Tymnos (Pérée rhodienne)}

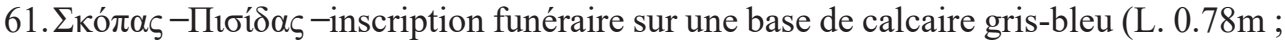
H. $0.28 \mathrm{~m}$; P. $0.78 \mathrm{~m}$ ) -basse époque hellénistique ou début d'époque impériale Chaviaras 1911, 66, nº 65 ; I. Peraia 219 ; I. Pérée 105 ; Bresson 1997, 122 ; LGPN

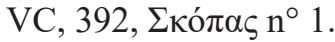

ABRÉVIATIONS

I. Peraia - W. Blümel, Die Inschriften der rhodischen Peraia, Bonn 1991.

I. Pérée - A. Bresson, Recueil des inscriptions de la Pérée rhodienne, Besançon-Paris 1991.

I. Selge $\quad-\quad$ J. Nollé, F. Schindler, Die Inschriften von Selge, Bonn 1991.

I. Tralles - F. B. Poljakov, Die Inschriften von Tralleis und Nysa, t. 1, Bonn 1989.

LGPN VA - A Lexicon of Greek Personal Names, vol. VA : T. Corsten, Coastal Asia Minor: Pontos to Ionia, Oxford, 2010.

LGPN VB - A Lexicon of Greek Personal Names, vol. VB : J.-S. Balzat, R. W. V. Catling, É. Chiricat, F. Marchand, Coastal Asia Minor : Caria to Cilicia, Oxford 2013.

$L G P N$ VC - A Lexicon of Greek Personal Names, vol. VC : J.-S. Balzat, R. W. V. Catling, É. Chiricat, T. Corsten, Inland Asia Minor, Oxford 2018.

SGDI - H. Collitz, E. Bechtel et al., Sammlung der griechischen Dialekt-Inschriften, 5 vols., Göttingen 1884-1915.

BIBLIOGRAPHIE

Badoud, N. (2017), Inscriptions et timbres amphoriques de Rhodes. Documents recueillis par le médecin et explorateur suédois Johan Hedenborg, Stockholm. 
Bean, G. E. (1970), The site of Etenna, Klio 52 : 13-16.

Berges, D., Nollé, J. (1996), Rundaltäre aus Kos und Rhodos, Berlin.

Biliotti, É., Cottret, A. (1881), L'île de Rhodes, Compiègne.

Blinkenberg, C. (1941), Lindos. Fouilles de l'acropole 1902-1914, t. II : Inscriptions, Berlin-Copenhague.

Boyxen, B. (2018), Fremde in der hellenistischen Polis Rhodos. Zwischen Nähe und Distanz, Berlin.

Bresson, A. (1997), Remarques préliminaires sur l'onomastique des esclaves dans la Rhodes antique, in : M. Moggi, G. Cordiano (éds.), Schiavi e dipendenti nell'ambito dell' 'oikos'e della 'familia'. Atti del XXII Colloquio GIREA Pontignano (Siena) 19-20 Novembre 1995, Pisa : 117-126.

Bricault, L. (2005), Recueil des inscriptions concernant les cultes isiaques, Paris.

Bru, H. (2017), La Phrygie Parorée et la Pisidie septentrionale aux époques hellénistique et romaine. Géographie historique et sociologie culturelle, Leiden-Boston.

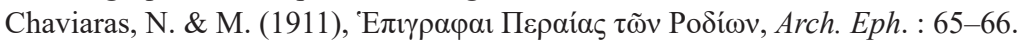

Chaviaras, N. \& M. (1913), Arch. Eph. : 3.

Dąbrowa, E. (1998), The Governors of Roman Syria from Augustus to Septimius Severus, Bonn.

Doni, C. (2009), The Pisidians: From Their Origin to Their Western Expansion, in : H. Bru, F. Kirbihler, S. Lebreton (éds.), L'Asie Mineure dans l'Antiquité : échanges, populations et territoires. Actes du colloque international de Tours (21-22 octobre 2005), Rennes : 213-227.

Ferrary, J.-L. (1985), La lex Antonia de Termessibus, Athenaeum 63 : 419-457.

Flacelière, R., Roussel, P. (1936), Bull. épigr. : 386.

Foucart, P. (1866a), Inscriptions inédites de l'île de Rhodes, $R A 13: 152-167$.

Foucart, P. (1866b), Inscriptions inédites de l'île de Rhodes, $R A 14$ : 328-338.

Foucart, P. (1886), Inscriptions de Rhodes, $B C H 10: 199-210$.

Foucart, P. (1889), Inscriptions de Rhodes, $B C H 13: 346-367$.

Foucart, P. (1969), Epidamia, in : C. Daremberg, E. Saglio, Dictionnaire des antiquités grecques et romaines, t. II.1, Graz : 660.

Fraser, P. M. (1977), Rhodian Funerary Monuments, Oxford.

Fraser, P. M., Bean, G. E. (1954), The Rhodian Peraea and Islands, London.

Garlan, Y. (1998), Les « fabricants » d'amphores, Topoi 8/2 : 581-590.

Goukowski, P. (2003), (éd.) Appien, Histoire romaine, Livre XII : La guerre de Mithridate, Paris.

Grace, V. R. (1934), Stamped Amphora Handles Found in 1931-1932, Hesperia 3 : 197-310.

Grace, V. R. (1956), Stamped Wine Jar Fragments: Small Objects from the Pnyx II, Hesperia Suppl. $10: 115-189$.

Grainger, J. D. (2002), The Roman War of Antiochos the Great, Leiden-Boston.

Hatzfeld, J. (1910), Inscriptions de Rhodes, BCH 34 : 242-248.

Hedenborg, J. (1854), Geschichte der Insel Rhodos, Ms.

Hicks, E. L. (1889), Inscriptions from Casarea, Lydae, Patara, Myra, JHS 10 : 46-85.

Hiller von Gaertringen, F. (1896), Inschriften aus Rhodos, MDAI(A) $21: 39-66$.

Hiller von Gaertringen, F. (1931), Rhodos, RE S 5 : 731-840.

Holleaux, M. (1931), La clause territoriale du traité d'Apamée (188 av. J.-C.), RÉG 44 : 304-319.

Holleaux, M. (1932), La clause territoriale du traité d'Apamée (188 av. J.-C.), RÉG 45 : 7-31.

Jacopi, G. (1932), Nuove epigrafi dalle Sporadi meridionali, Clara Rhodos 2 : 165-256.

Jones, C. P. (1992), Foreigners in a Hellenistic Inscription of Rhodes, Tyche 7 : 123-132.

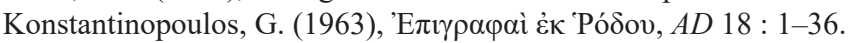

Konstantinopoulos, G. (1965), Chronika, $A D 20: 597$.

Konstantinopoulos, G. (1968), Chronika, $A D 23$ : 443-446.

Konstantinopoulos, G. (1969), Chronika, $A D 24: 470$.

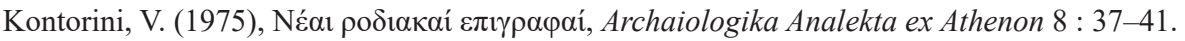

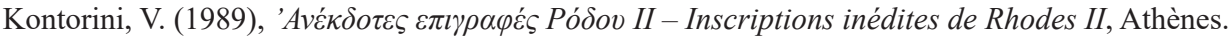

Kruse, B. (1934), Termessos, RE VA1 : 732-778.

Lambertz, M. (1907), Die griechischen Sklavennamen, t. I, Wien. 
Lambertz, M. (1908), Die griechischen Sklavennamen, t. II: Adjektiva als Sklavennamen, Wien.

Launey, M. (1987²), Recherches sur les armées hellénistiques, t. I-II, Paris.

Levick, B. (1967), Roman Colonies in Southern Asia Minor, Oxford.

Macridy, T. (1904), À travers les nécropoles sidoniennes, $R B i$ : 549-556.

Maiuri, A. (1921-1922), Un nuovo decreto di associazione a Rodi, ASAA 4-5 : 223-232.

Maiuri, A. (1925), Nuova Silloge Epigrafica di Rodi e Cos, Firenze.

Marangou, L., Papachristodoulou, I. (1993), Chronika, AD 48 : 538.

Masson, O. (1973), Les noms des esclaves dans la Grèce antique, in : Actes du Colloque de 1971 sur l'esclavage, Besançon 10-11 Mai 1971, Besançon : 9-23.

Migeotte, L. (2014), Les finances des cités grecques aux périodes classique et hellénistique, Paris.

Mitchell, S. (1991), The Hellenization of Pisidia, MedArch 4 : 119-145.

Morelli, D. (1956), Gli stranieri in Rodi, Studi Classici e Orientali 5 : 126-190.

Nicolaou, I. (2005), PAPHOS V: The Stamped Amphora Handles from the House of Dionysos, Nicosia.

Nicolaou, K. (1976), Archaeological News from Cyprus, 1974, AJA 80 : 361-375.

Nilsson, M. P. (1909), Timbres amphoriques de Lindos, Copenhague.

Nollé, J. (1984), Etenna. Ein Vorbericht, EA 3 : 142-156.

Nollé, J., Schindler, F. (1991), Die Inschriften von Selge, Bonn.

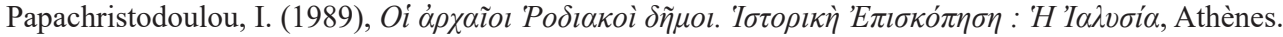
Perdrizet, P. (1904), Syriaca, RA 1904 : 234-244.

Peyras, J. (2010), Le siège de Rhodes par Mithridate (88 av. J.-C.), in : N. Faucherre, I. Pimouguet-Pédarros (éds.), Les sièges de Rhodes, de l'Antiquité à la période moderne, Rennes : 157-174.

Porro, G. G. (1916), Iscrizioni di Rodo, ASAA 2 : 125-131.

Pugliese-Carratelli, G. (1939-1940), Per la storia delle associazioni in Rodi antica, ASAA 17-18 : 147200.

Pugliese-Carratelli, G. (1952-1954), Supplemento epigrafico Rodio, ASAA 30-32 : 247-316.

Rey-Coquais, J.-P. (1976), Inscription grecque découverte à Ras Ibn Hani, AArchSyr 26 : 51-61.

Rey-Coquais, J.-P. (1978), Inscription grecque découverte à Ras Ibn Hani : stèle de mercenaires lagides sur la côte syrienne, Syria $55: 313-325$.

Robert, L. (1935), Notes d'épigraphie hellénistique. XLIII. Épitaphe d'un mercenaire à Sidon, $B C H$ 59 : 428-430.

Robert, L. (1936), Collection Froehner, t. I : Inscriptions grecques, Paris.

Robert, L. (1938), Études épigraphiques et philologiques, Paris.

Robert, L. (1954), La Carie. Histoire et géographie historique avec le recueil des inscriptions antiques. II. Le plateau de Tabai et ses environs, Paris.

Robert, J. \& L. (1955), Bull. épigr. : 265, n² 210.

Robert, J. \& L. (1958), Bull. épigr. : 289-290, nº 357.

Robert, L. (1963), Noms indigènes dans l'Asie Mineure gréco-romaine, Paris.

Robert, L. (1966a), Monnaies antiques en Troade, Paris-Genève.

Robert, J. \& L. (1966b), Bull. épigr. : 407, nº 298.

Robert, J. \& L. (1970), Bull. épigr. : 421, n 416.

Robert, J. \& L. (1972), Bull. épigr. : 438, n 322.

Robert, J. \& L. (1977), Bull. épigr. : 434-435, n 553.

Robert, J. \& L. (1982), Bull. épigr. : 359, $\mathrm{n}^{\circ} 244$.

Ruge, W. (1921), Selge, RE IIA1 : 1257.

Ruge, W. (1939), Oroandeis, RE XVIII, 1: 1130-1132.

Sacco, G. (1980), Su alcuni etnici di stranieri di Rodi, Rendic. Accad. Lincei 35 : 517-528.

Sartre, M. (2004²), L'Anatolie hellénistique, de l'Égée au Caucase, Paris.

Scrinzi, A. (1899), Iscrizioni greche inedite di Rodi, Venezia.

Sekunda, N. (2006), Hellenistic Infantry Reform in the 160's BC, Gdańsk.

Sherk, R. K. (1984), Rome and the Greek East, Cambridge.

Solin, H. (1996), Die stadtrömischen Sklavennamen. Ein Namenbuch, T. I-III, Stuttgart. 
Swoboda, H., Keil, J., Knoll, F. (1935), Denkmäler aus Lykaonien, Pamphylien und Isaurien, Wien. Syme, R. (1995), Anatolica. Studies in Strabo, ed. by A. Birley, Oxford.

Vidmann, L. (1969), Sylloge Inscriptionum Religionis Isiacae et Sarapiacae, Berlin.

Vollkommer, R. (éd.) (2001-2004), Künstlerlexikon der Antike, München-Leipzig.

Welles, C.B. $\left(1966^{2}\right)$, Royal Correspondence in the Hellenistic Period: A Study in Greek Epigraphy, Roma.

Will, E. (2003²), Histoire politique du monde hellénistique (323-30 av. J.-C.), t. II, Paris.

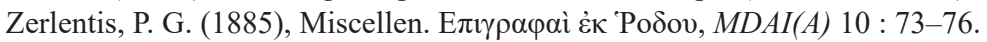

Zgusta, L. (1964), Kleinasiatische Personennamen, Prag.

Zgusta, L. (1984), Kleinasiatische Ortsnamen, Heidelberg. 